\title{
Numerical optimization of dc wire parameters for mitigation of the long range beam-beam interactions in High Luminosity Large Hadron Collider
}

\author{
K. Skoufaris ${ }^{*}$ \\ European Organization for Nuclear Research (CERN), CH-1211 Geneva 23, Switzerland; \\ Department of Physics, University of Crete, P.O. Box 2208, GR-71003 Heraklion, Greece; \\ and Institute of Theoretical and Computational Physics (ITCP), GR-71003 Heraklion, Greece \\ S. Fartoukh, Y. Papaphilippou®, A. Poyet@, A. Rossi॰, and G. Sterbini® \\ European Organization for Nuclear Research (CERN), CH-1211 Geneva 23, Switzerland \\ D. Kaltchev $(0$ \\ TRIUMF, 4004 Wesbrook Mall, Vancouver, B.C., Canada V6T 2A3
}

(Received 23 December 2020; accepted 6 July 2021; published 22 July 2021)

\begin{abstract}
Several configurations of the High Luminosity Large Hadron Collider, whose performance at collision is mainly limited due to the strong beam-beam long-range interactions, are studied in the presence of dc wire compensators. This analysis is based on analytical and numerical calculations where the main observables are the dynamic aperture (correlated to the beam lifetime) and the frequency map analysis. It is demonstrated that, with a proper optimization of the wire compensator parameters (distance from the beam and wire current) and without violating the machine protection restrictions, these long range beambeam interactions can be very well mitigated, making these scenarios viable and complementary with respect to the present HL-LHC baseline. The impact in the integrated luminosity and the operational flexibility gained are also presented.
\end{abstract}

DOI: 10.1103/PhysRevAccelBeams.24.074001

\section{INTRODUCTION AND MOTIVATION}

The counter rotating proton beams in the Large Hadron Collider (LHC) [1] share the same beam pipe at the insertion regions (IR), where the experiments are located. At the center of the detector, the beams cross each other and the particles undergo head-on (HO) collisions. Before and after these interaction points (IPs) and up to the separation dipoles (D1) the particles in the bunches interact with the electromagnetic field generated from the counter rotating ones every $12.5 \mathrm{~ns}$ (for bunch spacing $\Delta t=25 \mathrm{~ns}$ ) as it is illustrated in Fig. 1. The detrimental effect of these beambeam long range (BBLR) interactions on the particle dynamics was extensively studied in LHC and other colliders [2-8]. For the mitigation of the BBLR kicks, the use of electron lenses or current carrying wires (a technically simpler solution) was proposed and their effectiveness was tested in various machines like RHIC at BNL, SPS at CERN and TEVATRON at FNAL [9-12].

\footnotetext{
*kyriacos.skoufaris@cern.ch
}

Published by the American Physical Society under the terms of the Creative Commons Attribution 4.0 International license. Further distribution of this work must maintain attribution to the author(s) and the published article's title, journal citation, and DOI.
The use of wire compensators was initially proposed in an LHC note [13] and after the previously mentioned positive experimental results four wire demonstrators were installed at each high luminosity IP of the LHC (IP1 and IP5). Using these compensators in different experimental studies (that are accompanied by numerical ones) it was shown a significant improvement in beam lifetime [14-16]. These results encouraged using the wire demonstrators in the upcoming Run 3 of the LHC [17].

The effect of the BBLR interactions on the particles motion will be even more significant after the High Luminosity upgrade of the Large Hadron Collider (HL-LHC) [18-22].

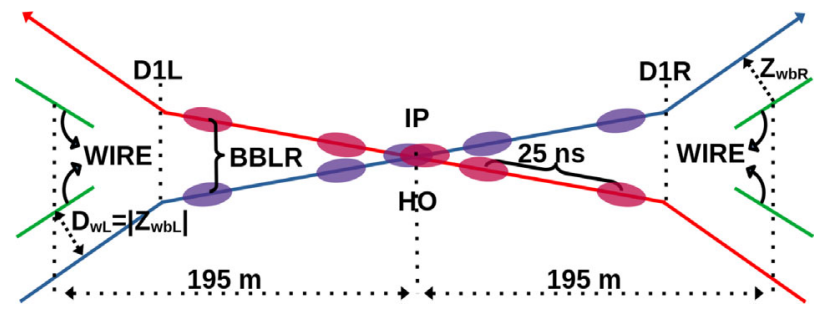

FIG. 1. Schematic representation (not to scale) for the head on collision, the beam beam long range kicks at the interaction regions and the reserved longitudinal position at the left and right of the IP1 and IP5 in the case of a future use of wire compensators in HL-LHC. 
TABLE I. HL-LHC baseline configuration at the start of collisions and the end of the luminosity leveling.

\begin{tabular}{|c|c|c|c|}
\hline Parameters & Symbol & $\begin{array}{l}\text { Start of } \\
\text { collisions }\end{array}$ & $\begin{array}{l}\text { End of luminosity } \\
\text { leveling }\end{array}$ \\
\hline Energy [GeV] & $E$ & 7000 & 7000 \\
\hline $\begin{array}{l}\text { Bunch } \\
\text { population [ppb] }\end{array}$ & $N_{p}$ & $2.2 \times 10^{11}$ & $1.2 \times 10^{11}$ \\
\hline $\begin{array}{l}\text { Normalized } \\
\quad \text { emittance }[\mu \mathrm{m}]\end{array}$ & $\varepsilon_{n x}=\varepsilon_{n y}$ & 2.5 & 2.5 \\
\hline Horizontal tune & $Q_{x}$ & 62.315 & 62.315 \\
\hline Vertical tune & $Q_{y}$ & 60.32 & 60.32 \\
\hline $\begin{array}{l}\text { Horizontal } \\
\text { chromaticity }\end{array}$ & $\xi_{x}$ & +15 & +15 \\
\hline Vertical chromaticity & $\xi_{y}$ & +15 & +15 \\
\hline $\begin{array}{l}\text { Beta function at } \\
\text { IP1 \& IP5 [cm] }\end{array}$ & $\beta^{\star}$ & 64 & 15 \\
\hline $\begin{array}{l}\text { Half crossing angle at } \\
\text { IP1 \& IP5 }[\mu \mathrm{rad}]\end{array}$ & $\frac{\Phi_{1 / 5}}{2}$ & 250 & 250 \\
\hline $\begin{array}{l}\text { Landau octupole } \\
\text { current }[\mathrm{A}]\end{array}$ & $I_{o}$ & -300 & -300 \\
\hline
\end{tabular}

The aim of this upgrade is to increase the luminosity (production rate of useful collisions), leading to an improvement of the machine discovery potential (faster reduction of the measurements statistical error). According to the baseline scenario [19] the leveled and integrated luminosity will be increased by a factor five and ten respectively over the LHC design values [23]. This improvement is mostly based on the brighter bunches (higher bunch population over smaller emittance), the stronger focusing at the IPs (smaller $\left.\beta^{*}\right)$ and the use of crab cavities [24-26]. The side effect of using brighter bunches for a given crossing angle is the stronger BBLR interactions that define the HL-LHC parameters of the current baseline configurations, shown in Table I.

Simulating the HL-LHC nominal scenario, the most severe degradation of the dynamic aperture (DA) and consequently of the beam lifetime [27] from the BBLR kicks can be observed at the end of the luminosity leveling where the optical beta at the IPs $\left(\beta^{*}\right)$ is minimum. This significant DA reduction is demonstrated in Fig. 2. The red curve corresponds to the baseline (nominal) case with only

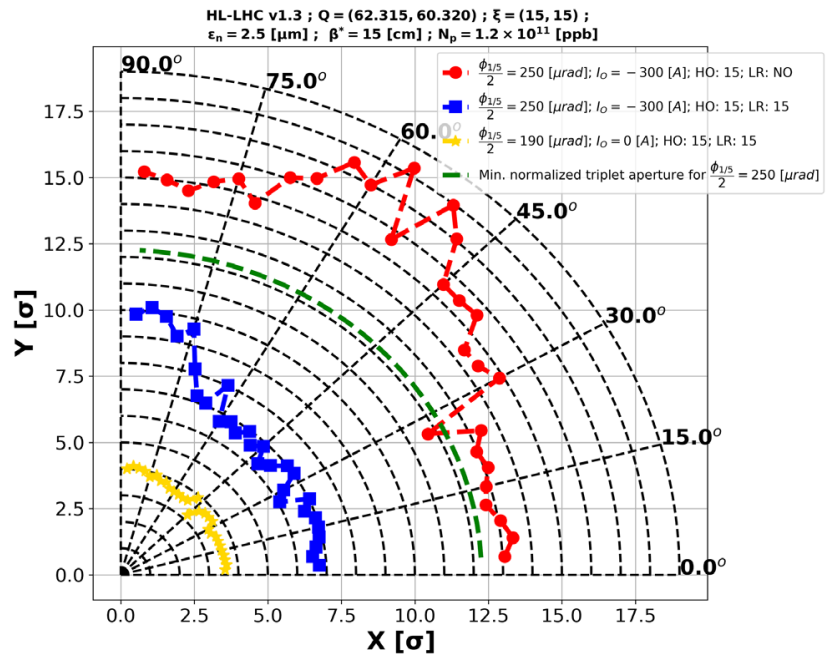

FIG. 2. Dynamic aperture vs crossing angles at the end of the luminosity leveling. For the case of only head-on collision at IP1 and IP5 is the red line, the case of head on plus BBLR kicks and $250 \mu \mathrm{rad}$ half crossing angle is the blue line, while with $190 \mu \mathrm{rad}$ is the yellow line. The minimum normalized triplet aperture for the HL-LHC baseline scenario is shown in green.

HO collision at IP1 and IP5, while the blue one to the case with $\mathrm{HO}$ collisions and 25 BBLR kicks per IP per side for IP1 and IP5 (20 BBLR kicks up to D1 separation dipole plus 5 inside D1). With green dashed line is indicated the minimum normalized triplet aperture of the HL-LHC which roughly corresponds to the minimum $\mathrm{DA}\left(\mathrm{DA}_{\min }\right)$ when BBLR collisions are not present (red curve). Even after systematic optimization studies of the parameters (working point, octupole current, etc.) [22], the $\mathrm{DA}_{\min }$ for the nominal scenario (blue curve) is only slightly above $6 \sigma$ and is reduced by $5.5 \sigma$ as compared to the $\mathrm{DA}_{\text {min }}$ of the case without BBLR kicks. Although a $6 \sigma$ minimum DA is considered to be enough for a good lifetime [28,29], there is not any margin for the baseline scenario for any unexpected detrimental effect like the impact of electron cloud (as the one limiting the lifetime during the last year of 2015-2018 run in LHC [30]). In addition, there is no flexibility to reduce the half crossing angle lower than $250 \mu \mathrm{rad}$ without

TABLE II. HL-LHC improved scenarios at the start of collisions and the end of the luminosity leveling.

\begin{tabular}{|c|c|c|c|c|}
\hline \multirow[b]{2}{*}{ Parameters } & \multicolumn{2}{|c|}{ Improved nominal scenario } & \multicolumn{2}{|c|}{ Improved ultimate scenario } \\
\hline & $\begin{array}{l}\text { Start of } \\
\text { collisions }\end{array}$ & $\begin{array}{l}\text { End of luminosity } \\
\text { leveling }\end{array}$ & $\begin{array}{l}\text { Start of } \\
\text { collisions }\end{array}$ & $\begin{array}{l}\text { End of luminosity } \\
\text { leveling }\end{array}$ \\
\hline Energy $[\mathrm{GeV}]$ & 7000 & 7000 & 7000 & 7000 \\
\hline Bunch population [ppb] & $2.2 \times 10^{11}$ & $1.2 \times 10^{11}$ & $2.2 \times 10^{11}$ & $1.52 \times 10^{11}$ \\
\hline Normalized emittance $[\mu \mathrm{m}]$ & 2.5 & 2.5 & 2.5 & 2.5 \\
\hline $\begin{array}{l}\text { Beta function at } \\
\text { IP1 \& IP5 [cm] }\end{array}$ & 64 & 15 & 64 & 15 \\
\hline $\begin{array}{l}\text { Half crossing angle at } \\
\text { IP1 \& IP5 }[\mu \mathrm{rad}]\end{array}$ & 190 & 190 & 200 & 200 \\
\hline
\end{tabular}


sacrificing the limit of $6 \sigma$ minimum DA. After the halving of the crab cavities number in HL-LHC, the maximum crabbing angle is limited to $190 \mu \mathrm{rad}$. Simulating a scenario with $190 \mu \mathrm{rad}$ half crossing angle at IP1 and IP5 (needed for a full crabbing compensation at the IPs), the DA degradation is significant and lies between 3-4 $\sigma$ (yellow curve of Fig. 2). Therefore, any performance improvement from a perfect $\mathrm{HO}$ collision or any other benefits from the crossing angle reduction are prohibited due to the strong BBLR interactions.

In order to address these limitations, the use of wire compensators in HL-LHC nominal round optics $\left(\beta_{x}^{*}=\beta_{y}^{*}\right)$ configuration is studied. Based on analytical and numerical optimization studies, the findings in [31] are extended and new operational schemes of the HL-LHC called improved nominal and improved ultimate (Table II) are found and presented in following sections. These new schemes, improve significantly the performance and the operational flexibility of the collider without violating the present machine protection restrictions.

\section{ANALYTICAL CALCULATIONS}

\section{A. Limitations at the BBLR compensation}

For the study of the long range beam-beam interactions, the weak-strong approximation is used [32,33]. According to this, the particles in the "weak" beam interact with the electromagnetic field generated by the charge distribution of the "strong" beam while the latter beam is not affected by the charge distribution of the former one. Neglecting any optics error, the quantity studied in this paper is the integrated Lorentz force and its expression as well the

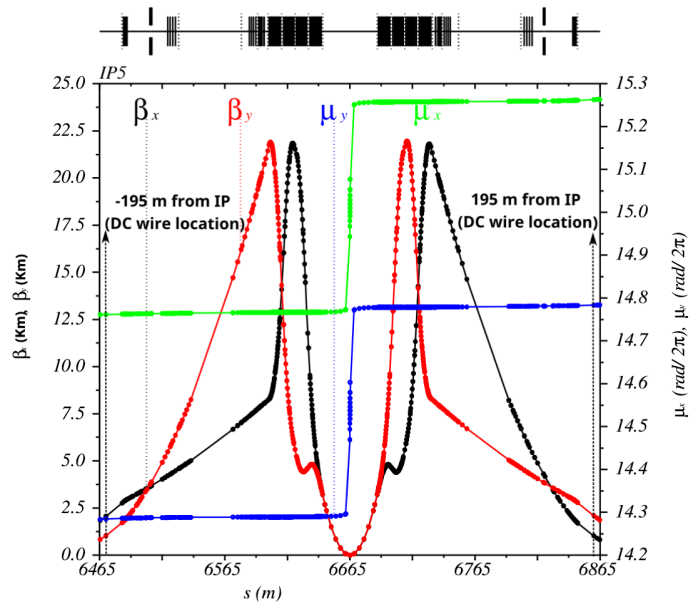

(a)

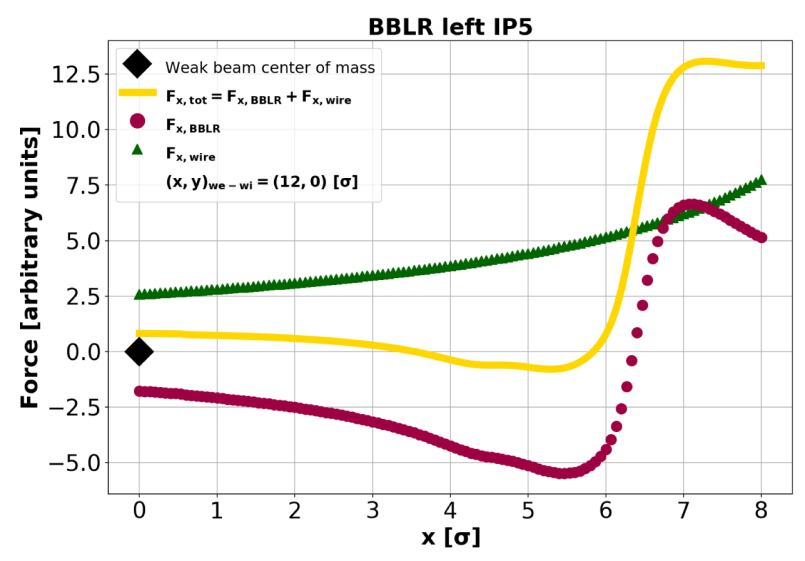

(b)

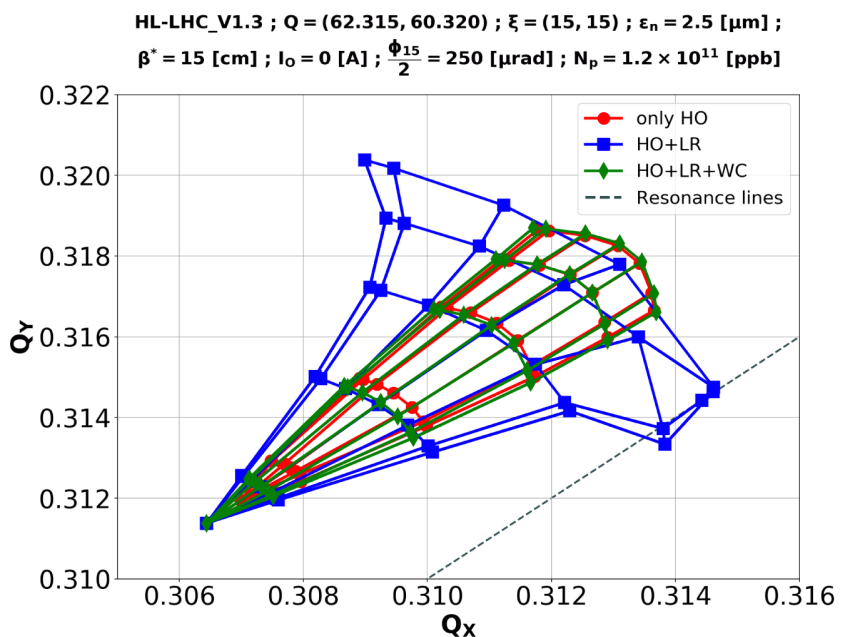

(c)

FIG. 3. (a) Beta function $\left(\beta_{x}, \beta_{y}\right)$ and phase advance $\left(\mu_{x}, \mu_{y}\right)$ at the IR5 for $\beta^{*}=15 \mathrm{~cm}$. (b) At the left of the IP5 the Lorentz force generated from a wire (green triangles) that is placed at $12 \sigma$ from the weak beam (black rhombus), the force from a composed nonlinear map that include the BBLR kicks and the optics between them (magenta circles) and the total force from the composed non-linear map and the dc wire (yellow solid line) at the crossing plane. (c) Footprint plots for three different configurations: only head on collisions (red), head on \& BBLR kicks (blue), head on \& BBLR kicks \& wire compensation (green). 
electromagnetic fields generated by a strong bunch $(\mathcal{B})$ and a dc wire $\left(\mathcal{B}_{w}\right)$ are shown in the Appendix. As can be seen from the Fig. 12, the $\mathcal{B}$ and $\mathcal{B}_{w}$, away from their source, are quite similar in the dependence of test particles position. Based on this similarity the dc wires are proposed as a good compensation device for the BBLR kicks. The correlation of the dynamics between the BBLR kick and the force generated by the dc wire was extensively studied in work [5].

Although a BBLR compensation device is not included in the current operational scenario of the HL-LHC, ring sections that are $\sim 10 \mathrm{~m}$ long and are located at $\sim 195 \mathrm{~m}$ left and right of the IP1 and IP5 are reserved for a potential use of dc wires (Fig. 1). These longitudinal positions are right after the IRs (close to the Q4s) and thus, the phase advance up to the first BBLR kick right before the IPs is small (less than $0.07 \mathrm{rad}$ ) as can be seen in Fig. 3(a). The benefit from the small phase advance is the capability to compensate locally the resonance driving terms (RDT) that are driven by the BBLR kicks. On the other hand, there are some limitations that must be applied at the wire current $I_{w}$ and its transverse distance from the weak beam $D_{w}=\left|\mathcal{Z}_{w b}\right|$ $\left(\mathcal{Z}_{w b}\right.$ is a position vector in complex plain discussed in Appendix. The most restrictive one is coming from the machine protection considerations and in particular from the aperture of the elements and tertiary collimators hierarchy. At the start of collisions, the transverse distance of the wire from the weak beam $\left(D_{w}\right)$ must be larger than 18 (17.4) $\sigma$ for the baseline (ultimate) scenario and drop to $10.4 \sigma$ at the end of the luminosity leveling for both the baseline and ultimate scenarios [20]. The restrictions to the $I_{w}$ are coming from the physical properties of the wire. Based on the current R\&D [34] for a wire that could be used in the HL-LHC, the maximum current for a wire module with $L_{w}=3 \mathrm{~m}$ and cross section at the scale of millimeters is $I_{w} \sim 450 \mathrm{Am}$. In addition to these limitations, the strong variations of the beta function [Fig. 3(a)] at long range beam-beam interactions (drift from the IP to Q1 and inside the inner triplet) increase the complexity of the problem. As a result, the BBLR separation as well the BBLR kicks are not the same at each encounter, with the absolute kicks right before and after the IPs to be the strongest ones. Taking into account this variation, the force at the horizontal $(x)$ crossing plane at the left of IP5 from a composed nonlinear map that include the BBLR kicks and the optics between them is plotted with magenta circles in Fig. 3(b). In the same figure, the force from a wire compensator is also plotted with green triangles. As can be seen from the yellow solid line (total force) the detrimental effect from the BBLR kicks can be mitigated quite well up to $6 \sigma$. In this showcase example, the wire is

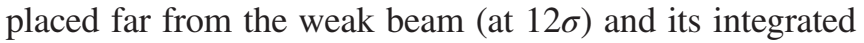
current $\left(I_{w}\right)$ is appropriately tuned in order to minimize the force from the BBLR kicks up to $6 \sigma$.

\section{B. Compensation of the tune spread with amplitude generated by the BBLR kicks}

Analytical formulas that describe the resonance driving terms (RDT) and the tune spread with amplitude (TSA) that are driven by the BBLR and the dc wire kicks are developed in different works. Some of them, in order to extract formulas in closed forms, assume wire like BBLR kicks [31,35] while others, for simplicity, use round bunches $\left(\sigma_{x}=\sigma_{y}\right)$ for all the BBLR kicks [33]. These assumption are good only for large distances between the strong and the weak beam. A different approach is followed in $[6,36,37]$ where the scalar potential that describe the BBLR field is used but the resulting expressions involve integrals over the bunch distribution that should be calculated at the location of the BBLR interactions. In a recent work [38], analytical expressions for the RDT and the TSA are derived without any of the aforementioned approximations-limitations and therefore are the ones used in this paper. On top of these formulas, some extra simplifications can be obtained in the case of the HL-LHC (and LHC) optics. Because the crossing plane is rotated by $90^{\circ}$ between the IP1 and IP5 (e.g., for IR1 $\left.\mathcal{Z}_{w b}\right|_{\text {IR1 }}=\mathrm{i} y_{w b}$ and for IR5 $\left.\mathcal{Z}_{w b}\right|_{\mathrm{IR} 5}=x_{w b}$ ), the coefficients $c_{u}$ [Eq. (A3)] for two wires (or two wire-like BBLR kicks) that are located at IP1 and IP5 with the same transverse distance, satisfy the equation $\left.c_{u}\right|_{\text {IR } 1}=-\left.c_{u}\right|_{\text {IR5 }}$ for $u=4 m+1$ and $m=0,1,2, \ldots$ (quadrupolar, decapolar, etc., components). On the other hand, for the multipoles with $u=4 m+3$ and $m=0,1,2, \ldots$ (octupolar, decahexapolar, etc.) $\left.c_{u}\right|_{\mathrm{IR} 1}=$ $\left.c_{u}\right|_{\text {IR5 }}$. For these wire/wirelike configurations and because the HL-LHC optical functions at the IR1 and IR5 are almost identical, the tune shift and the first order $\mathrm{TSA}_{\chi}$ that is generated by the $u=4 m+1$ multipoles is selfcompensated while the first order $\mathrm{TSA}_{\chi}$ from the $u=$ $4 m+3$ multipoles adds up. Therefore the octupolar component is the first non-self-compensated one that contribute at first order to the $\operatorname{TSA}_{\chi}$.

In order to evaluate how well the dc wires can mitigate the tune spread with amplitude $\left(\mathrm{TSA}_{\chi}\right)$ generated by the BBLR kicks, HL-LHC footprints with (a) only HO, (b) HO and BBLR interactions, and (c) HO, BBLR interactions and wire compensators at IP1 and IP5 are plotted in Fig. 3(c). Simulating the baseline scenario at the end of the luminosity leveling with zero Landau octupoles current and zero rf cavities voltage, the footprint without BBLR kicks (only $\mathrm{HO}$ ) and the one with BBLR kicks (HO + LR) are plotted with red and blue color, respectively. Including de wires fully compliant with the machine protection restrictions (wire distance is at least $10.4 \sigma$ from the weak beam), the tune spread generated by the BBLR interactions is well mitigated as can be seen from the green footprint $(\mathrm{HO}+\mathrm{LR}+\mathrm{WC})$ in Fig. 3(c). The very good agreement between the green and red footprints indicates that the wires can control well the tune spread with amplitude induced by the BBLR kicks. For the calculation of the wire integrated 
current the analytical formulas in [38] and the selfcompensation conditions are used while the footprints are calculated with the MAD-X code [39].

\section{NUMERICAL OPTIMIZATION OF THE DC WIRE FOR BEST COMPENSATION OF THE BBLR INTERACTIONS}

It has been shown that if the wires are placed at a longitudinal position with optical beta ratio $\left(\beta_{x, w} / \beta_{y, w}\right)$ close to 2 or 0.5 , all the leading order RDTs can be minimized with the appropriate wire current and transverse position in $[31,35]$. This result was obtained using the wire like approximation for the BBLR kicks, alongside with a few small simplifications, like the negligible phase advance and dispersion at the IRs, exact antisymmetric optics between the left and right of the IP and between the two beams at the same side of the IP. However, based on the reserved location for the dc wires and the formulas for the RDTs minimization, the wires' transverse distance $D_{w}$ and their integrated current $I_{w}$, for the working scenario described in Table I with antisymmetric optics between the left (L) and the right (R) sides of the IPs $\left(\frac{\beta_{x, w L}}{\beta_{y, w L}}=r^{*^{2}} \frac{\beta_{y, w R}}{\beta_{x, w R}}\right.$ with $\left.r^{*}=\frac{\beta_{y}^{*}}{\beta_{x}^{*}}\right)$ and negligible phase advance between the BBLR kicks, are $D_{w}=7.36 \mathrm{~mm}=8.85 \sigma_{w R 1}$ and $I_{w}=122 \mathrm{Am}$. As already mentioned, this transverse distance is presently not compatible with the machine protection aspects (i.e., collimation aperture hierarchy). In order to estimate the order of magnitude of the wire configurations that compensate the BBLR interactions (lifetime-dynamic aperture improvement) and respect the machine protection restrictions $\left(D_{w}>18 \sigma\right.$ at the start of collisions and $D_{w}>10.4 \sigma$ at the end of the luminosity leveling), a set of different DA scans for a variety of wire and lattice parameters such as the wire integrated current $\left(I_{w}\right)$, the wire transverse distance from the weak beam $\left(D_{w}\right)$, the Landau octupole current $\left(I_{o}\right)$ and the working tune $\left(Q_{x}\right.$, $Q_{y}$ ) were performed. The considered quality factor in all the scans is the difference between the minimum dynamic aperture $\left(\mathrm{DA}_{\min }\right)$ of a lattice with wire compensators from a lattice without wire, $\Delta \mathrm{DA}_{\min }=\mathrm{DA}_{\min }$ (with wire)$\mathrm{DA}_{\text {min }}$ (nowire). The best wire configurations with $D_{w}>$ $18 \sigma$ at the start of collisions and $D_{w}>10.4 \sigma$ at the end of luminosity leveling are called best conditional configurations (best conditional $\mathrm{DA}_{\min }$ ) and are indicated with green asterisks in the following plots. In all the simulations, four compensators (one for each side of the IP1 and IP5) are used and are longitudinally placed $195 \mathrm{~m}$ away from IP center. In addition, the four wires are in the IP crossing plane and parallel to the weak beam as it is shown in Fig 1. Since the optical functions are not the same for the four wire compensators, their configurations $\left(D_{w}\right.$ and $\left.I_{w}\right)$ are not exactly the same. In the following DA scan plots, only the configuration at the right of IP1 $\left(D_{w R 1}\right.$ and $\left.I_{w R 1}\right)$ are displayed since the wire at the right of IP1 (left of IP5) is always closer to weak beam $\left(D_{w R 1}\right.$ is always the smallest distance from the weak beam). For the $I_{w}-D_{w}$ scans, the central configuration is the one that compensates the octupolar components of the BBLR field. In all the studies, the linear chromaticity is kept constant at 15 units in both plains. The HO and BBLR kicks at IP2 and IP8 are not included in the simulations since they are significant weaker than the ones in IP1 and IP5. No magnet imperfections are considered and the tracking simulations (element by element tracking) are done with SIXTRACK code [40], where an equivalent thin lens lattice is used.

\section{A. HL-LHC baseline and ultimate scenario at the end of the luminosity leveling}

Starting with the HL-LHC baseline scenario at the end of the luminosity leveling with half crossing angle at IP1 and IP5 $\frac{\Phi_{1 / 5}}{2}=250 \mu \mathrm{rad}$, Landau octupoles set at $I_{o}=-300 \mathrm{~A}$ for partial compensation of the leading order octupolelike $\mathrm{TSA}_{\chi}$ from the BBLR kicks (as it is shown in [41] for LHC) and optimized working point $Q=(62.315,60.32)$ [22], the $\mathrm{DA}_{\min }$ without the use of dc wires is $\mathrm{DA}_{\min }$ (no wire) = $6.1 \sigma$ as shown in Fig. 4(a). Making use of the dc wires, the $\mathrm{DA}_{\min }$ can be improved up to $\Delta \mathrm{DA}_{\min }=0.7 \sigma$ with configurations that comply with the machine protection restrictions $\left(D_{w}>10.4 \sigma\right)$. The extra gain at the $\mathrm{DA}_{\min }$ can provide the margin for a flawless operation even in the presence of unexpected detrimental effects (like electron cloud formation). The most powerful configuration (smaller $D_{w}$ and larger $I_{w}$ ) of the dc wire demonstrators embedded in tertiary collimators of the LHC, is shown with a green square. Its large radius $(3 \mathrm{~mm})$ combined with the limiting $I_{w}=350 \mathrm{Am}$ provides only a marginal DA improvement, and this points out to the necessity of new dedicated hardware.

In order to better appreciate the dc wires impact on different particles, the DA values for different phase angles (different initial conditions) are plotted in Fig. 4(b). The different wire configurations that guarantee $\mathrm{DA}_{\min } \geq$ $\mathrm{DA}_{\min }$ (no wire) are plotted with different colors while the case without compensators is plotted in black. As can be seen, the DA improvement across the different angles can be quite more significant than the $\Delta \mathrm{DA}_{\min }$. The largest DA improved with wire compensators can be seen in those phase angles (indicated with gray shade) in which DA are reduced most with the BBLR kicks. The results from the wire configurations that provide the best conditional $\mathrm{DA}_{\text {min }}$ are shown with green asterisks and can give DA values close to the ones resulted from the ideal wire configurations (red dots) which give the best $\mathrm{DA}_{\min }$ (this ideal configuration is not complaint from any $D_{w}$ restriction).

An extra complication in the effort to find the best wire configurations is coming from the PACMAN bunches. In the various filling schemes [42] of the HL-LHC [43], the bunches are separated by $25 \mathrm{~ns}$. However, different trains (set of bunches) in the filling schemes are separated by a 


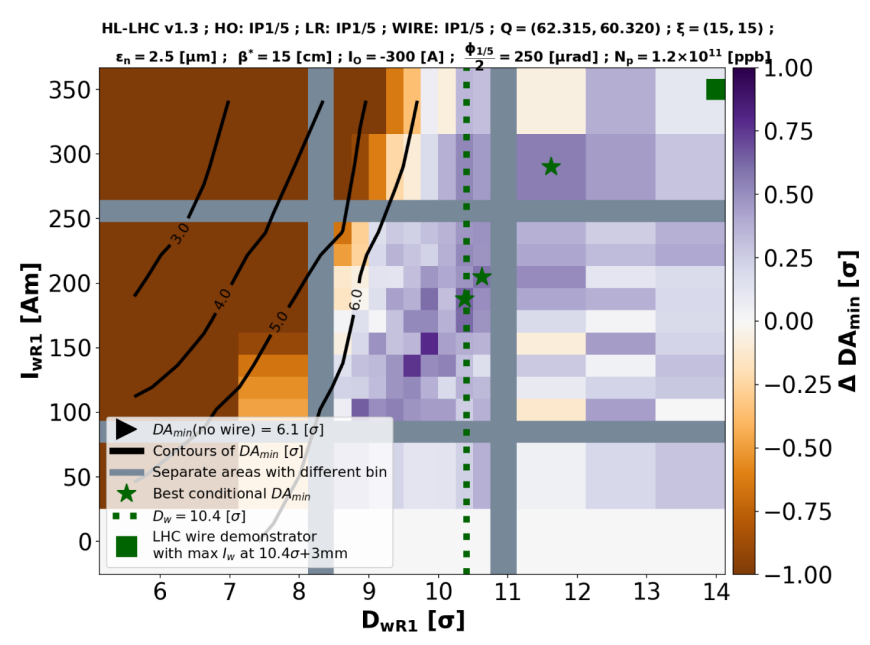

(a)

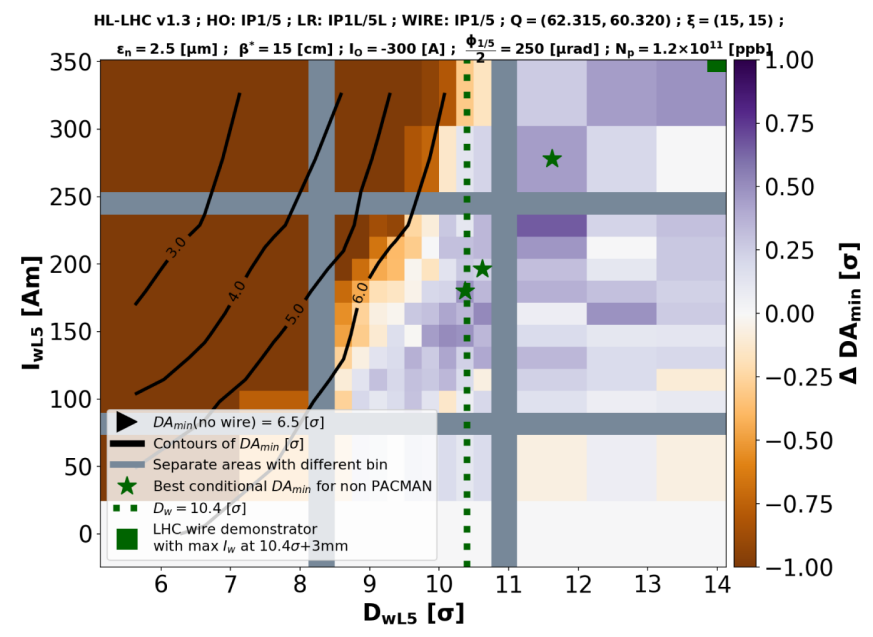

(c)

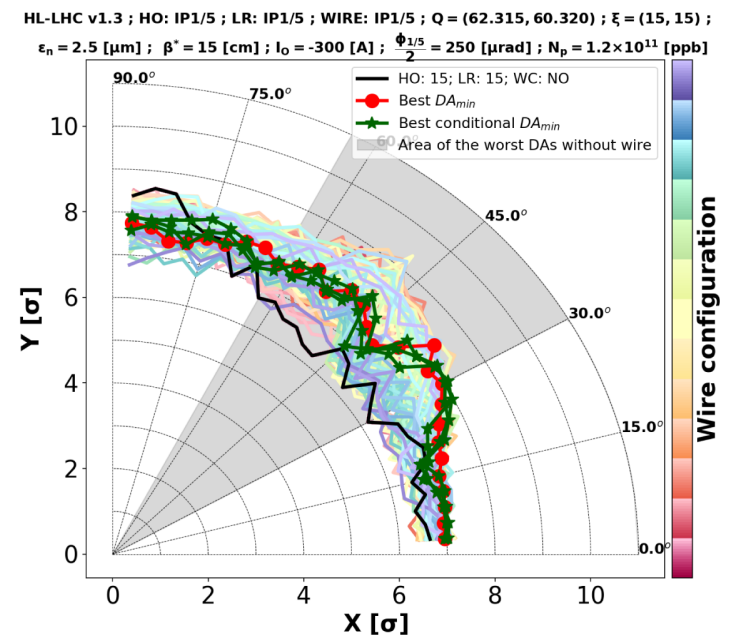

(b)

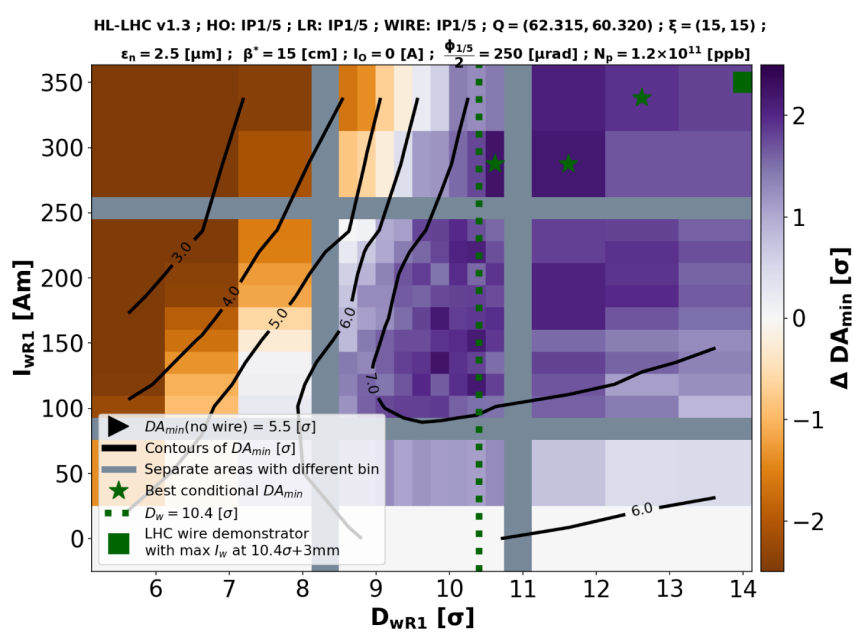

(d)

FIG. 4. HL-LHC nominal scenario at the end of the luminosity leveling. (a) The minimum DA difference from the case without wire $\left(\Delta \mathrm{DA}_{\min }\right)$ for different dc wire configurations and (b) DA values vs angles for the wire configurations that guarantee $\mathrm{DA}_{\text {min }} \geq \mathrm{DA}_{\text {min }}$ (no wire). (c) $\triangle \mathrm{DA}_{\min }$ for PACMAN bunches using different wire configurations. (d) $\Delta \mathrm{DA}_{\min }$ for different wire configurations and zero Landau octupoles $\left(I_{o}=0 \mathrm{~A}\right)$.

larger bunch space according to the rise times of the different injection and extraction kickers, the circumference of the injectors and the length of the abort gap. Due to this inhomogeneous distribution, the number of the BBLR kicks that are experienced by the different bunches is not the same for all of them. The ones located at the tails of the train experience less BBLR interactions and are called PACMAN bunches. The number of the BBLR interactions experienced by a PACMAN bunch ( $N_{\text {PACMAN }}$ ) lies in the range $N_{\mathrm{BBLR}}-1 \geq N_{\mathrm{PACMAN}} \geq \frac{N_{\mathrm{BBLR}}}{2}$ where $N_{\mathrm{BBLR}}$ is the full set of BBLR kicks at the interaction regions. The least amount of BBLR kicks is experienced by the first bunch of the first train after the abort gap and the last bunch of the last train before the abort gap. Given that the BBLR and wire kicks are similar and that the phase advance between them is negligible, the total kick from $N_{\text {PACMAN }}$ BBLR interactions and a dc wire that is adjusted to compensate the full set of BBLR kicks $\left(N_{\mathrm{BBLR}}\right)$ is equivalent to the kick from $N_{\text {BBLR }}-N_{\text {PACMAN }}$ BBLR interactions. This partial compensation always reduces the number of the equivalent BBLR interactions from $N_{\text {PACMAN }}$ to $N_{\text {BBLR }}-N_{\text {PACMAN }} \leq$ $N_{\text {PACMAN }}$ (the particle dynamics is less perturbed with the use of dc wire even for PACMAN bunches). Using the nominal scenario and simulating a PACMAN bunch that experience only the left BBLR interactions of the IR1 and IR5 (the last bunch of the last train before the abort gap with minimum number of BBLR kicks $N_{\text {PACMAN }}=\frac{N_{\text {BBLR }}}{2}$ ), the $\Delta \mathrm{DA}_{\min }$ for the same wire configurations as before (baseline scenario with the full set of BBLR kicks) can be seen in Fig. 4(c). The $\mathrm{DA}_{\min }$ not only is not degraded with the good wire configurations but it is actually improved compared to the case without compensation. Focusing 
on the configurations indicated with green asterisks that are the ones that give the best conditional $\mathrm{DA}_{\min }$ at the baseline scenario with full set of BBLR kicks, the $\mathrm{DA}_{\min }$ is up to $7.1 \sigma$ ( $0.5 \sigma$ improvement over the case without wire). Of course the ideal scenario would be to use a wire compensator with modulated $I_{w}$ according to different bunches but this increases the technological complexity of the compensator [44].

An import parameter that should be considered in these simulations is the Landau octupole current. During collisions the tune spread generated from the head on interactions [red footprint in Fig. 3(c)] can guarantee the stability of the colliding bunches $[45,46]$ and so as already mentioned, the Landau octupoles can be used for the mitigation of the leading order octupole-like tune spread with amplitude generated by the BBLR kicks. In order to calculate the compensation effect related only to the dc

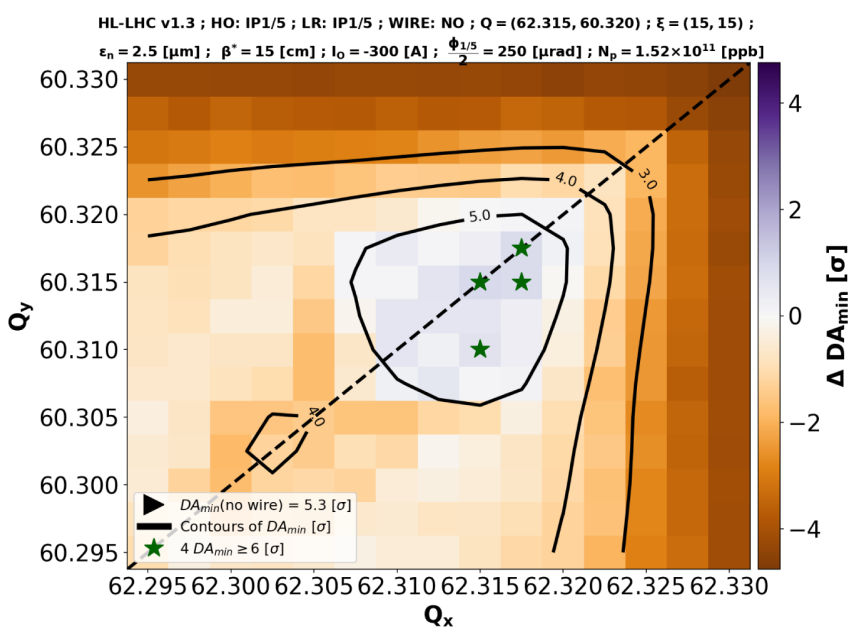

(a)

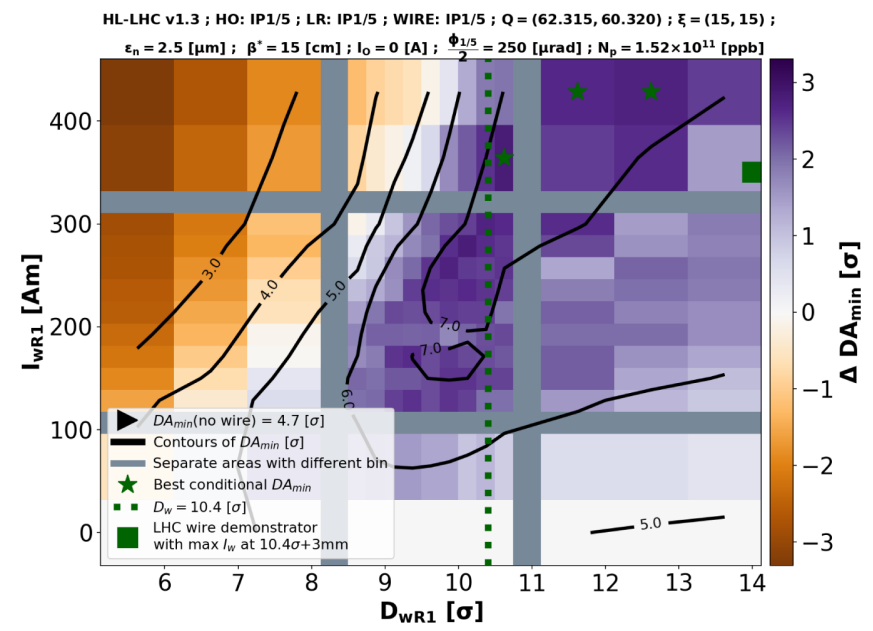

(c) wires, the DA scans for the nominal scenario at the end of the luminosity leveling but with zero Landau octupoles $\left(I_{o}=0 \mathrm{~A}\right)$ are repeated and the $\Delta \mathrm{DA}_{\min }$ is presented in the Fig. 4(d). The $\mathrm{DA}_{\min }$ without dc wire is less than $6 \sigma\left(\mathrm{DA}_{\min }\right.$ (no wire) $=5.5 \sigma$ ) but with the use of dc wires there are many configurations with $D_{w}>10.4 \sigma$ that guarantee $\mathrm{DA}_{\min }>6 \sigma$. The best of them, that are indicated with green stars, can guarantee $\mathrm{DA}_{\min }$ up to $7.8 \sigma(2.3 \sigma$ improvement over the case without wire). From the results in Figs. 4(a) and 4(d), it is clear that the DA gain is higher when only wire compensators are used (zero octupole current). The reason for this difference, is the overcompensation of the octupolelike component generated by the BBLR kicks in the case with non zero octupole current. Despite this overcompensation, the different configurations with $\mathrm{DA}_{\min } \geq 6 \sigma$ and $D_{w}>10.4 \sigma$ in Fig. 4(a), indicates that some choices of the octupole current can be destructive

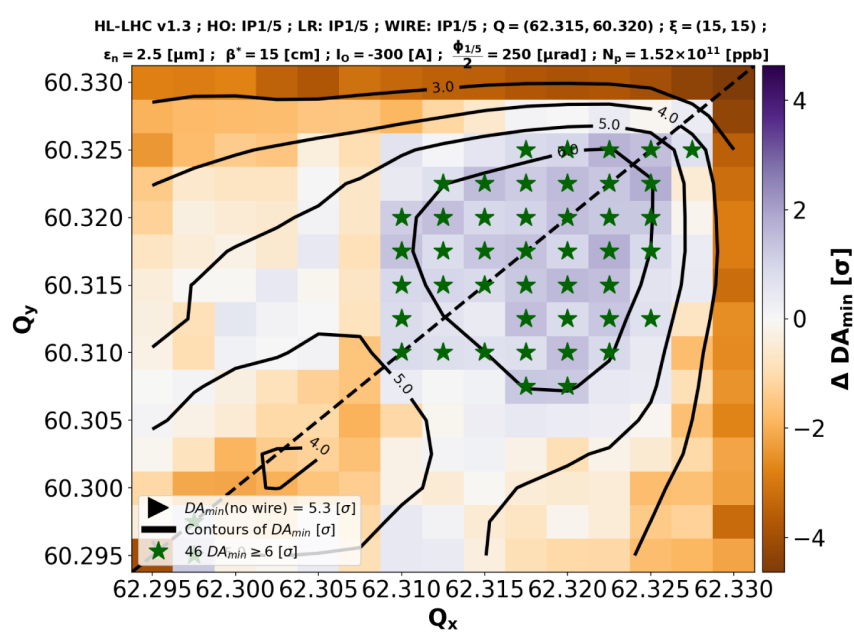

(b)

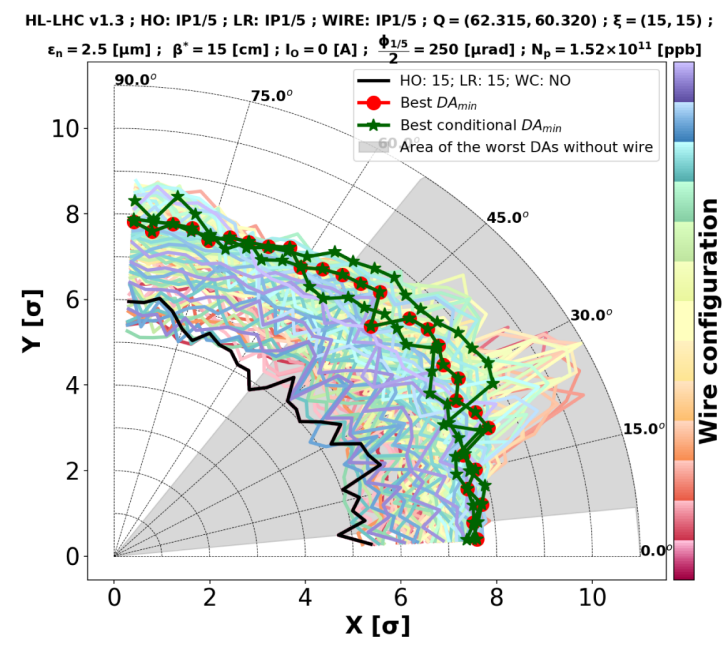

(d)

FIG. 5. HL-LHC ultimate scenario at the end of luminosity leveling. (a) and (b) $\Delta \mathrm{DA}_{\min }$ from different working points for the case without and with wire compensator, respectively, (c) $\Delta \mathrm{DA}_{\min }$ for different wire configurations and (d) DA values vs angles for wire configurations that guaranty $\mathrm{DA}_{\min } \geq \mathrm{DA}_{\min }$ (no wire). 
for the wire performance but not catastrophic. On the other hand, with an appropriate choice of the octupole current, the dc wire performance can be enhanced as it is shown in a following study.

In order to evaluate the compensation capabilities of the dc wires, the Landau octupoles are set to zero $\left(I_{o}=0 \mathrm{~A}\right)$ in the following studies, unless it is stated otherwise.

At the ultimate scenario of the HL-LHC, the luminosity is leveled at $7.5 \times 10^{34} \mathrm{~cm}^{-2} \mathrm{~s}^{-1}$ (instead of $5 \times$ $10^{34} \mathrm{~cm}^{-2} \mathrm{~s}^{-1}$ at the nominal scenario) and therefore the bunch population will be higher $\left(N_{p}=1.52 \times 10^{11} \mathrm{ppb}\right.$ at the end of leveling). Because of the increased bunch population, the BBLR kicks are stronger and so the

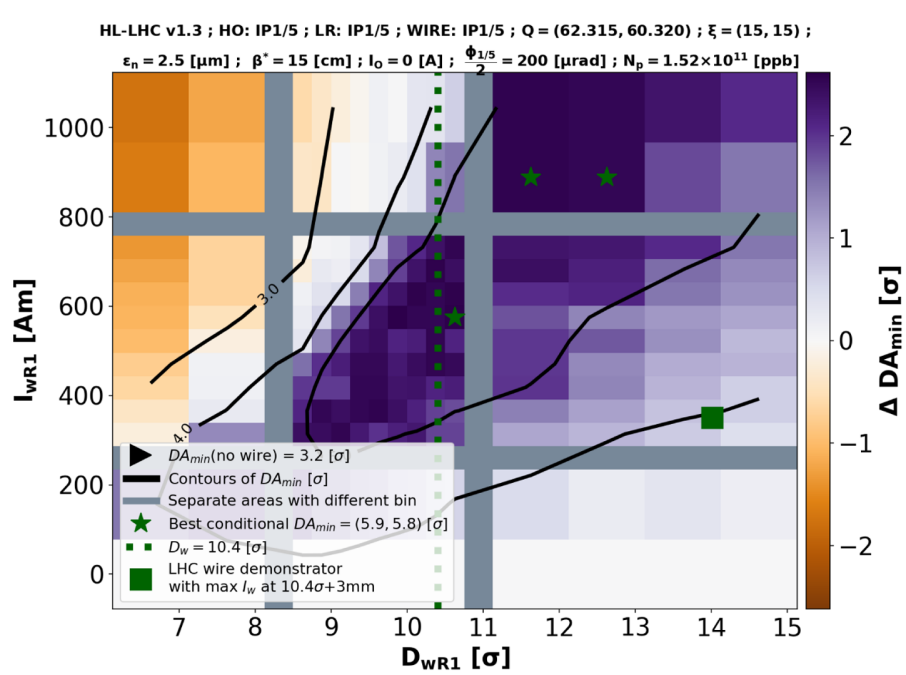

(a)

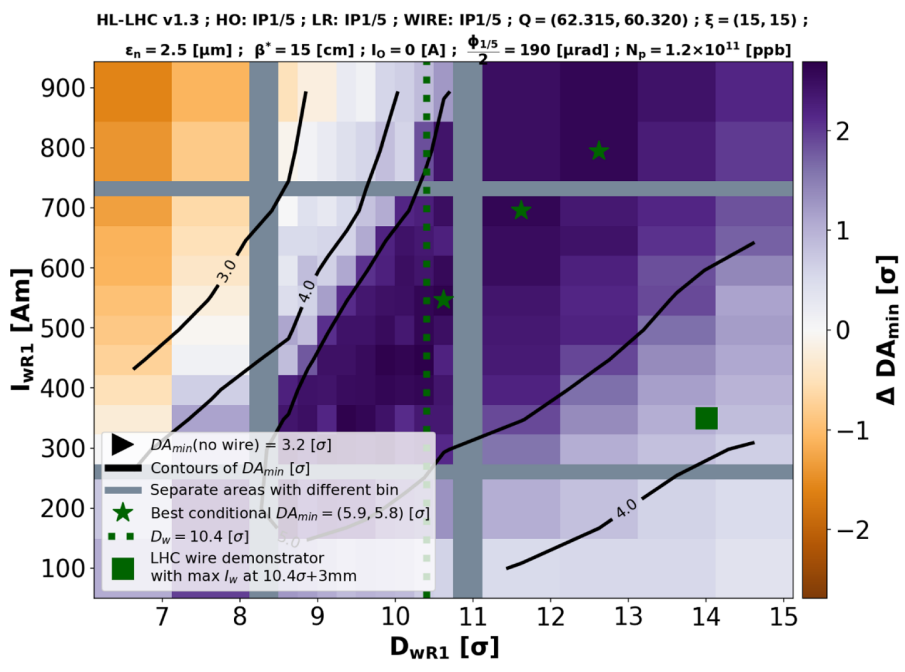

(c)
$\mathrm{DA}_{\min }$ degradation is more significant as compared to the one of the nominal scenario. As can be seen in Fig. 5(a), at the end of the luminosity leveling where the BBLR effect is stronger, there is no good working point at least 0.005 (due to coupling) from the diagonal that guaranty $\mathrm{DA}_{\min } \leq 6 \sigma$, despite the partial BBLR compensation with the use of the Landau octupoles. There is only one good operational configuration that does not overlap or is close to the diagonal, however since it is only one and it is below the diagonal, it can not be used. In fact, the beams are planned to be injected in the HL-LHC with a tune above the diagonal and working points below the diagonal may not be possible due to e-cloud tune-shift mainly at injection and

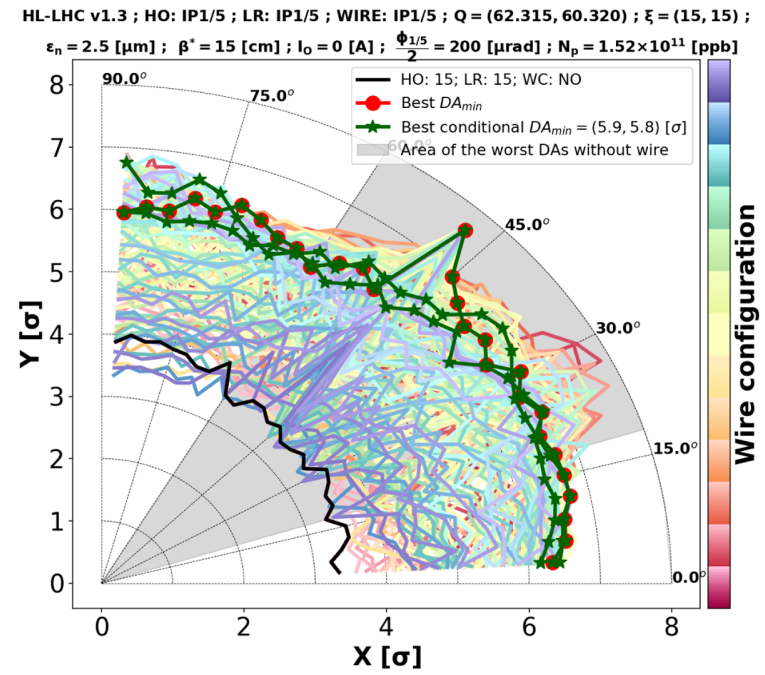

(b)

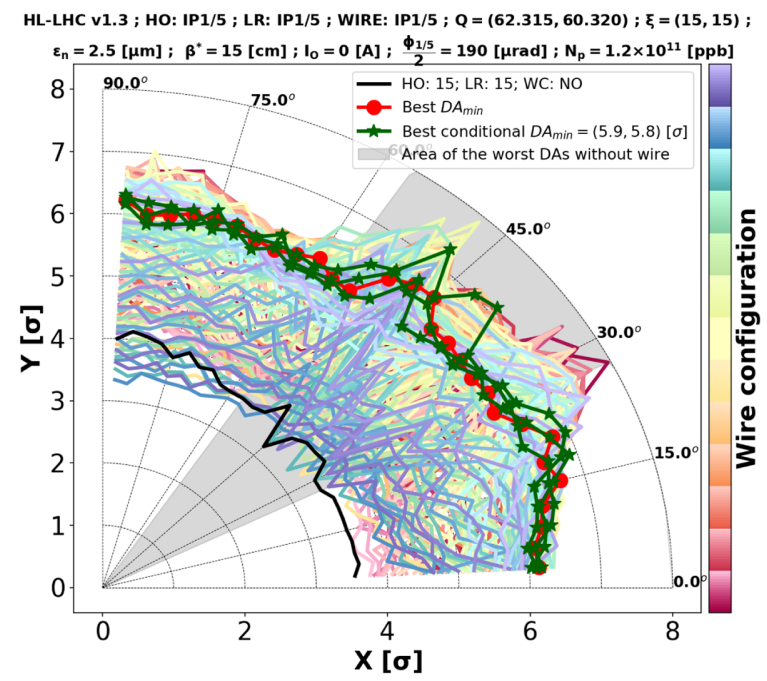

(d)

FIG. 6. HL-LHC at the end of luminosity leveling. (a) and (b) with half crossing angle at IP1 and IP5 $200 \mu \mathrm{rad}$ and $N_{p}=$ $1.52 \times 10^{11} \mathrm{ppb}$ the $\Delta \mathrm{DA}_{\min }$ for different wire configurations and the DA values vs angles for the wire configurations that guaranty $\mathrm{DA}_{\text {min }} \geq \mathrm{DA}_{\text {min }}$ (no wire), respectively. (c) and (d) with half crossing angle at IP1 and IP5 $190 \mu \mathrm{rad}$ and $N_{p}=1.2 \times 10^{11}$ ppb the $\Delta \mathrm{DA}_{\min }$ for different wire configurations and the DA values vs angles for the wire configurations that guaranty $\mathrm{DA}_{\min } \geq \mathrm{DA}_{\min }$ (no wire), respectively. 
the strong losses while crossing the diagonal resonance line. Keeping the same lattice and adding wire compensators with appropriate transverse positions $\left(D_{w}>10.4 \sigma\right)$ and $I_{w}$, a large "island" with good working points $\left(\mathrm{DA}_{\min } \geq 6 \sigma\right)$ appears, see Fig. 5(b). Therefore, the wire compensators can also guarantee a large set of good working points that improve the machine flexibility and can make the ultimate scenario of the HL-LHC feasible. In order to find the best parameters for the dc wire, a set of $\mathrm{DA}_{\min }$ scans are performed and the results are displayed in Fig. 5(c). Again there is a large set of good configurations $\left(\mathrm{DA}_{\min } \geq 6 \sigma\right)$ compatible with the machine protection limitations. The best of them are indicated with green asterisks and gives $\mathrm{DA}_{\min }$ up to $7.6 \sigma$ (2.9 $\sigma$ improvement

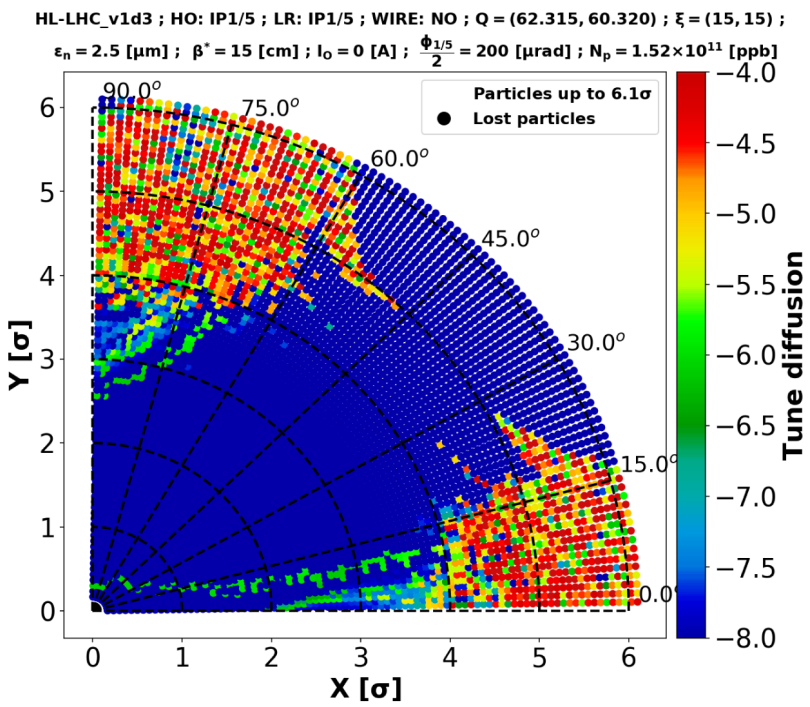

(a)

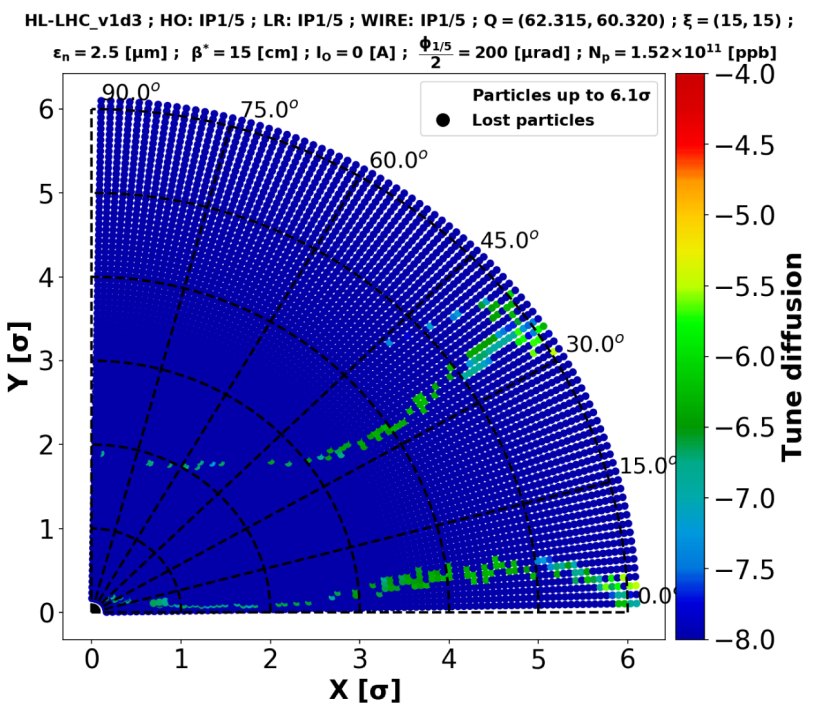

(c) over the case without wire). In Fig. 5(d) a more detailed view of the DA over the different angles for the wire configurations that guarantee $\mathrm{DA}_{\min } \geq \mathrm{DA}_{\min }$ (no wire) can be seen. There are many configurations that are significantly above the black line which corresponds to the case without wire. As before, the DA values of the best conditional configurations (indicated with green asterisks) are very similar to the ones from the configurations that give the best $\mathrm{DA}_{\min }$ (red dots).

\section{B. Improved HL-LHC scenarios}

In the following, we show several scenarios whose performance, thanks to wire compensator, become acceptable. These scenarios result from the reduction of the

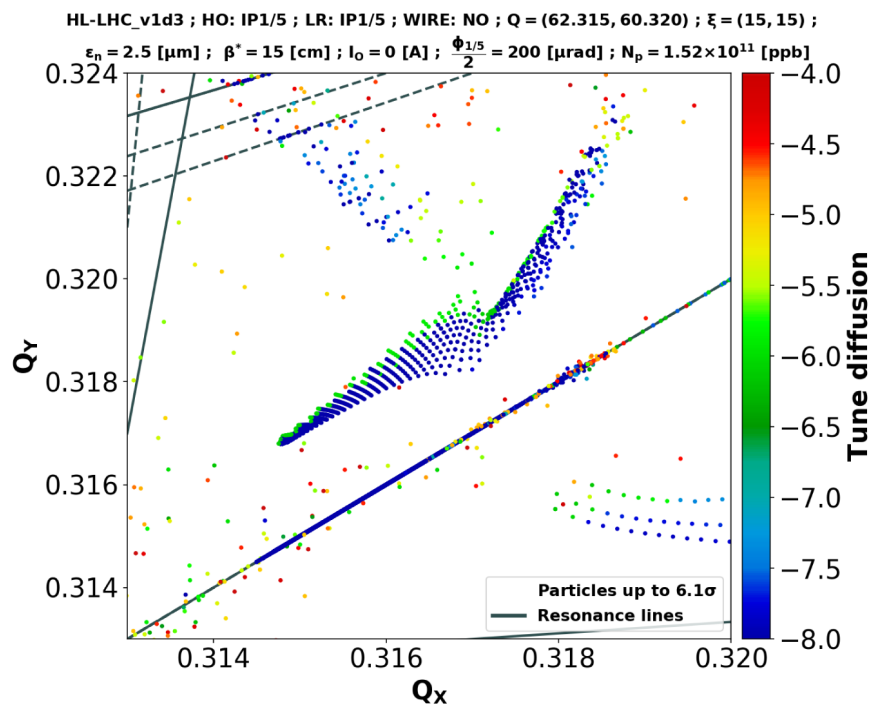

(b)

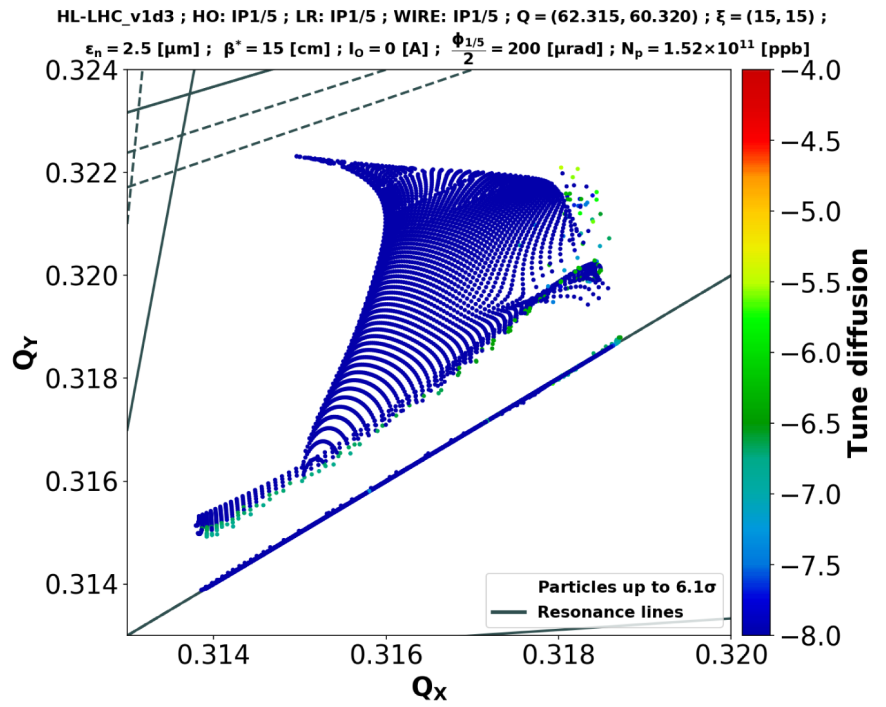

(d)

FIG. 7. HL-LHC at the end of luminosity leveling with half crossing angle at IP1 and IP5 $200 \mu \mathrm{rad}$ and $N_{p}=1.52 \times 10^{11} \mathrm{ppb}$. (a) and (c) for particles up to $6.1 \sigma$ the tune diffusion of the cases without and with wire compensator, respectively. (b) and (d) for particles up to $6.1 \sigma$ the footprint and the tune diffusion of the cases without and with wire compensator, respectively. 
crossing angle at the IP1 and IP5 $\left(\Phi_{1 / 5}\right)$. In this way the collider performance and its flexibility are improved as compared to the ones from the current nominal and ultimate scenarios. With smaller crossing angle, the luminosity leveling can be extended, increasing thereby, the integrated luminosity per fill. In addition, a smaller crossing angle allows the reduction of the crab cavity voltage [24-26] without degrading the integrated luminosity. Furthermore, the magnets (dipolar corrector) strength that are assisting in the crossing bumps, the required mechanical aperture, the heat load as well the integrated radiation dose coming from the debris produced at the IPs and mainly deposited in the triplet (final focus quadrupoles) [47] can be reduced. The goal in these studies was again to find the dc wire

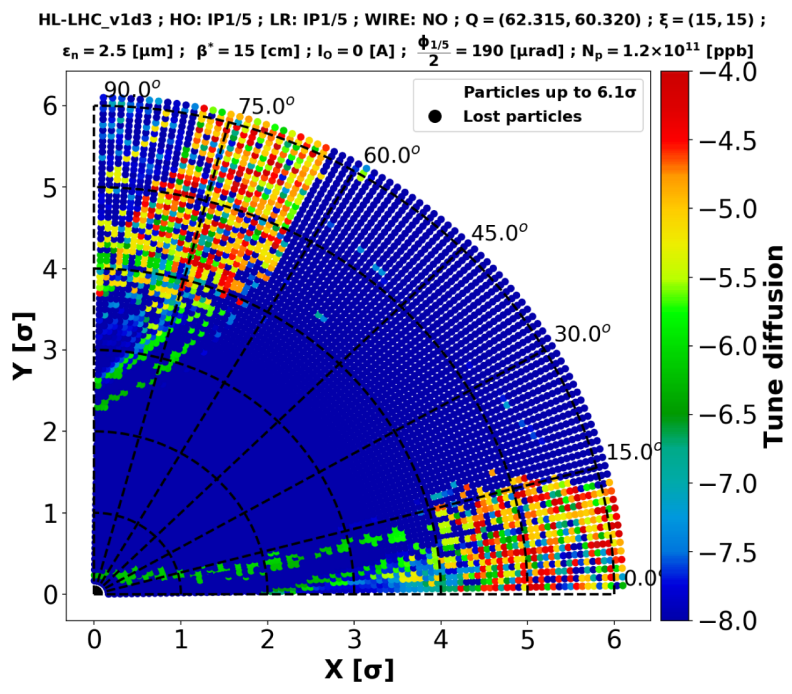

(a)

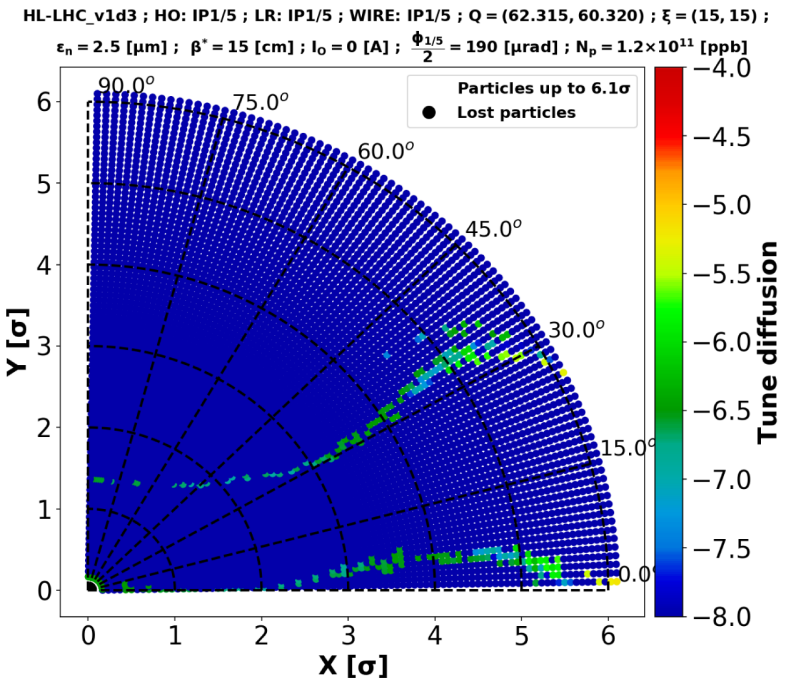

(c) configurations, within collimation constrains, that guarantee $\mathrm{DA}_{\min }$ close or above $6 \sigma$.

The improved operational scenarios $\left(\mathrm{DA}_{\min } \simeq 6 \sigma\right)$ that can be achieved with the use of wire compensators in HLLHC are the improved ultimate one with half crossing angle at IP1 and IP5 $\frac{\Phi_{1 / 5}}{2}=200 \mu \mathrm{rad}$ and the improved nominal scenario with half crossing angle at IP1 and IP5 $\frac{\Phi_{1 / 5}}{2}=190 \mu \mathrm{rad}$. Performing DA scans at the end of the luminosity leveling, the results for the improved ultimate $\left(\frac{\Phi_{1 / 5}}{2}=200 \mu \mathrm{rad}\right.$ and $\left.N_{p}=1.52 \times 10^{11} \mathrm{ppb}\right)$ and for the improved nominal scenarios $\left(\frac{\Phi_{1 / 5}}{2}=190 \mu \mathrm{rad}\right.$ and $N_{p}=1.2 \times 10^{11} \mathrm{ppb}$ ) can be seen in Figs. 6(a) and 6(c), respectively. For both cases, the $\mathrm{DA}_{\min }$ without wire is

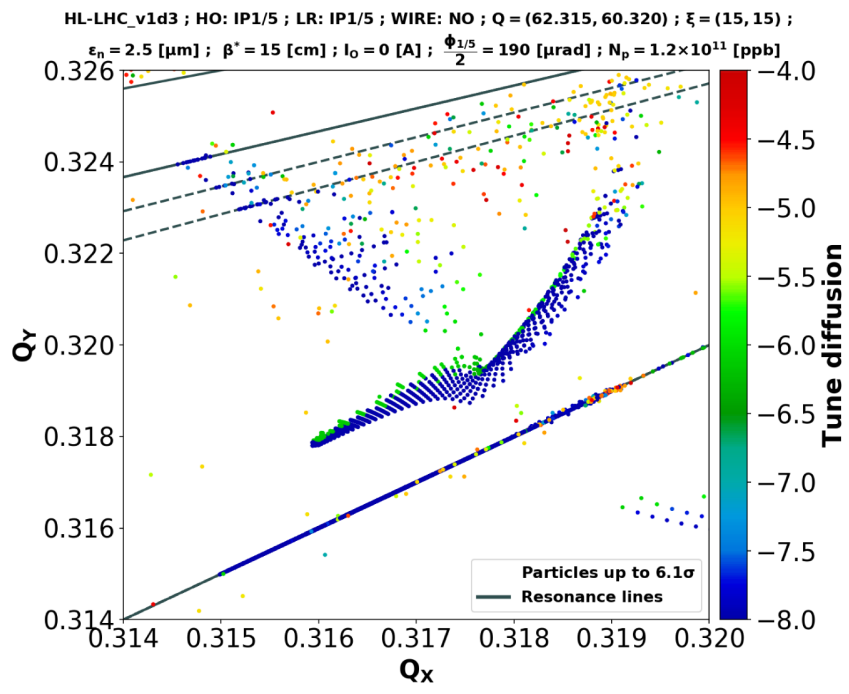

(b)

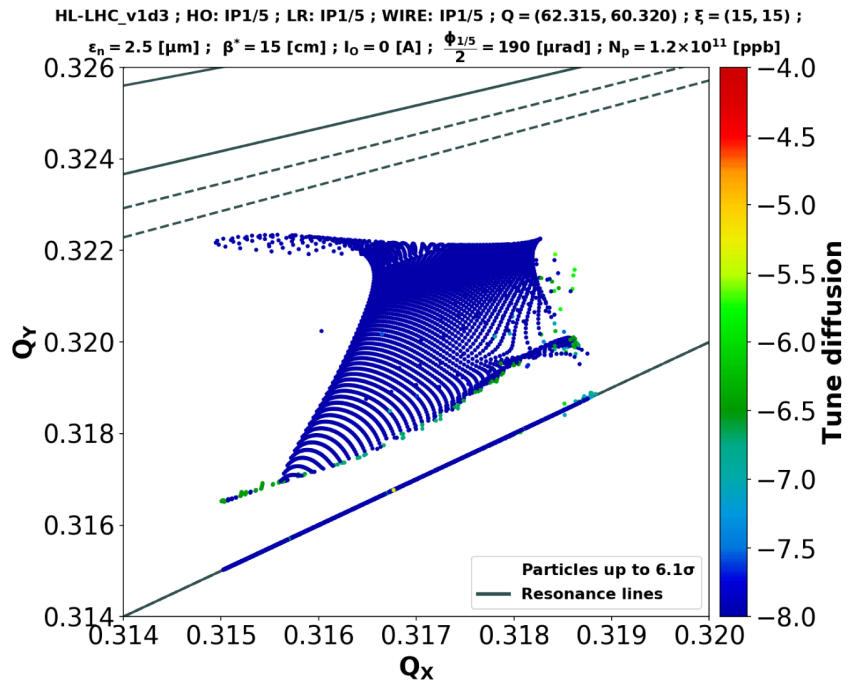

(d)

FIG. 8. HL-LHC at the end of luminosity leveling with half crossing angle at IP1 and IP5 $190 \mu \mathrm{rad}$ and $N_{p}=1.2 \times 10^{11} \mathrm{ppb}$. (a) and (c) for particles up to $6.1 \sigma$ the tune diffusion of the cases without and with wire compensator, respectively. (b) and (d) for particles up to $6.1 \sigma$ the footprint and the tune diffusion of the cases without and with wire compensator, respectively. 
below $3.5 \sigma\left(\mathrm{DA}_{\min }\right.$ (no wire) $\left.<3.5 \sigma\right)$ which is catastrophic for the beam lifetime. With the use of wire compensators, the best conditional configurations $\left(D_{w}>10.4 \sigma\right)$ can give a $\mathrm{DA}_{\min }$ up to $5.9 \sigma(2.7 \sigma$ improvement over the case without wire) which can guarantee a very good lifetime for the beams. In these improved scenarios, the DA restriction $\left(\mathrm{DA}_{\min } \geq 6 \sigma\right.$ ) can be slightly relaxed in order to improve the integrated luminosity. A more detailed overview of the DA for different angles can be seen in Figs. 6(b) and 6(d) for the improved ultimate and improved nominal scenarios, respectively. As in the previous cases, the results from the best conditional configurations are indicated with green asterisks and are mostly above $6 \sigma$. These curves are very close to the ones with the best $\mathrm{DA}_{\min }$ (red dots).

The beneficial impact of the dc wires at the performance oriented scenarios can be also seen from a frequencies map analysis [48]. In these improved scenarios the boosted BBLR interactions enhance the machine resonances and thus, the KAM tori [49-51] are destroyed leading to more irregular motions and increased tune diffusion. An estimate of tune diffusion in the frequency space is given by the formula $\log _{10}\left[\sqrt{\left(Q_{x, i n}-Q_{x, f i}\right)^{2}+\left(Q_{y, i n}-Q_{y, f i}\right)^{2}}\right]$ where the tune $\left(Q_{x, \text { in }}, Q_{y, \text { in }}\right)$ is calculated from the tracking data of the first 5000 turns and the tune $\left(Q_{x, f i}, Q_{y, f i}\right)$ from the data of the next 5000 turns. For the calculation of the $Q_{x}$ and $Q_{y}$ tunes, the NAFF algorithm [48,52] is used. The results for the improved ultimate and the improved nominal at the end of the leveling are presented in Fig. 7 and in Fig. 8, respectively. For particles up to $6.1 \sigma$, the use of dc wires [Figs. 7(c) and 8(c)] reduce significantly the strong tune diffusion seen at the case without compensators [Figs. 7(a) and 8(a)]. This compensatory effect can be also seen from the footprints up to $6.1 \sigma$ in Figs. 7(b), 7(d), 8(b), and $8(\mathrm{~d})$. In the absence of wire compensators [Figs. 7(b)

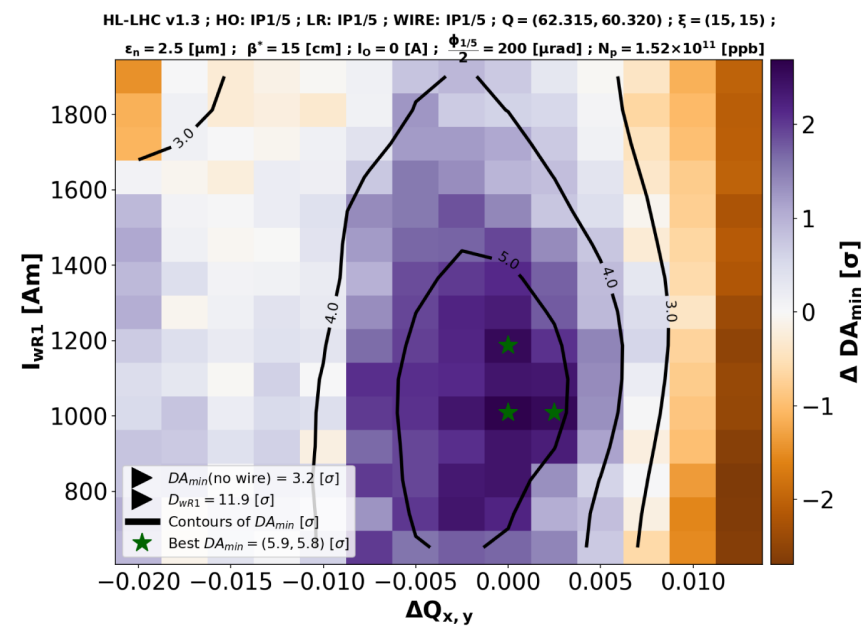

(a) and 8(b)] the tune spread is larger and the footprint extensively deformed. On the other hand, in the presence of dc wires [Figs. 7(d) and 8(d)] the tune diffusion is minimized and the footprint is compensated.

\section{Operational flexibility of the improved scenarios}

From the two improved scenarios, the one that guarantees larger integrated luminosity is the improved ultimate one. For that reason, the flexibility in the working point $(Q)$ selection and Landau octupole current $\left(I_{o}\right)$ with wire configurations that are at least $1.5 \sigma$ away from the tertiary collimators $\left(D_{w} \geq 11.9 \sigma\right)$ is studied. For different working points with constant fractional tune split $Q_{y}-Q_{x}=0.005$ and for different $I_{w}$ the $\Delta \mathrm{DA}_{\min }$ can be seen in Fig. 9(a). Within the $5.5 \sigma$ contour are many good configurations that guarantee $\mathrm{DA}_{\text {min }}$ close to $6 \sigma$. The best of them are indicated with green asterisks and can give a $\mathrm{DA}_{\min }$ up to $5.9 \sigma$. Even in this "pushed" scenario with fixed $D_{w}$ there is a flexibility in the operational tune and in $I_{w}$. For the other test, the tune is kept constant $\left(Q_{x}=62.315, Q_{y}=60.32\right)$ and the varying parameters are the Landau octupole current $\left(I_{o}\right)$ and the $I_{w}$. The resulting $\Delta \mathrm{DA}_{\min }$ is plotted in Fig. 9(b). By using both arc octupoles ( 150 A) and wires ( 350 Am), different wire configurations with $\mathrm{DA}_{\min } \geq 6 \sigma$ are found and are indicated with green asterisks. The DA improvement is up to $3 \sigma$ in a very "pushed" scenario and actually for an area that is relatively large ( $\pm 20-30 \mathrm{~A})$. In the case without wire compensators $\left(I_{w}=0 \mathrm{Am}\right)$ there are no configurations that guarantees $\mathrm{DA}_{\min } \geq 6 \sigma$. In addition to that, the FMAs of Figs. 7 and 8 demonstrates that the correction affects not only the linear but also the nonlinear tune spread with amplitude quite a lot.

In order to validate the impact of the dc wires also at the beginning of the leveling $\left(\beta^{*}=64 \mathrm{~cm}\right)$, DA scans for

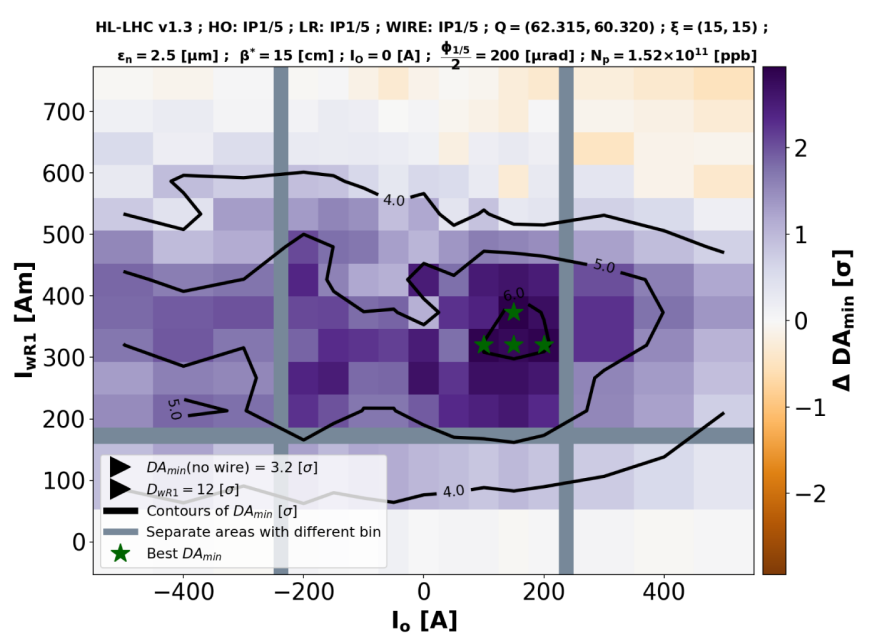

(b)

FIG. 9. HL-LHC at the end of luminosity leveling. (a) with half crossing angle at IP1 and IP5 $200 \mu \mathrm{rad}$ and $N_{p}=1.52 \times 10^{11} \mathrm{ppb}$ the $\Delta \mathrm{DA}_{\min }$ for different wire integrated current and working tunes. (b) with half crossing angle at IP1 and IP5 $200 \mu \mathrm{rad}$ and $N_{p}=$ $1.52 \times 10^{11} \mathrm{ppb}$ the $\Delta \mathrm{DA}_{\min }$ for different wire integrated current and different Landau octupole current. 


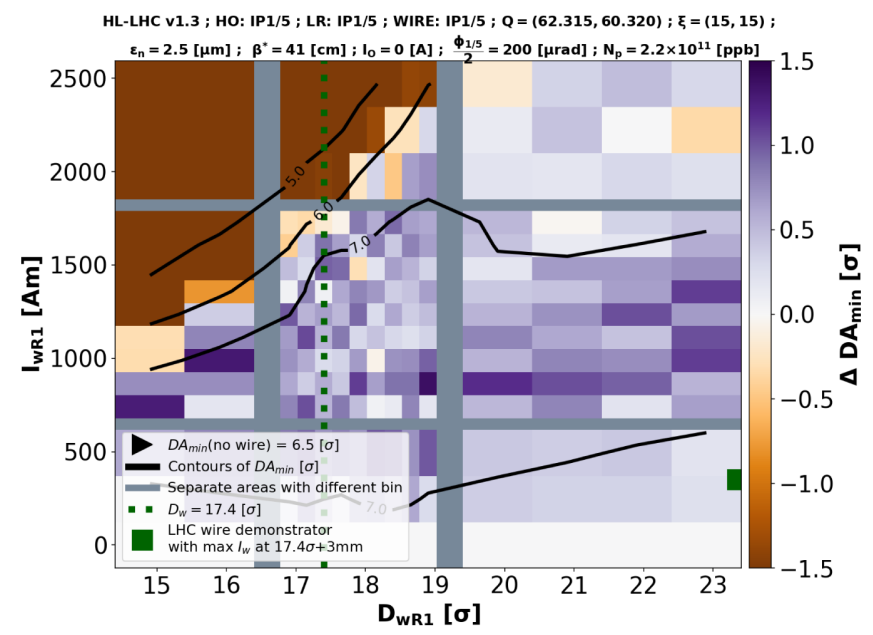

(a)

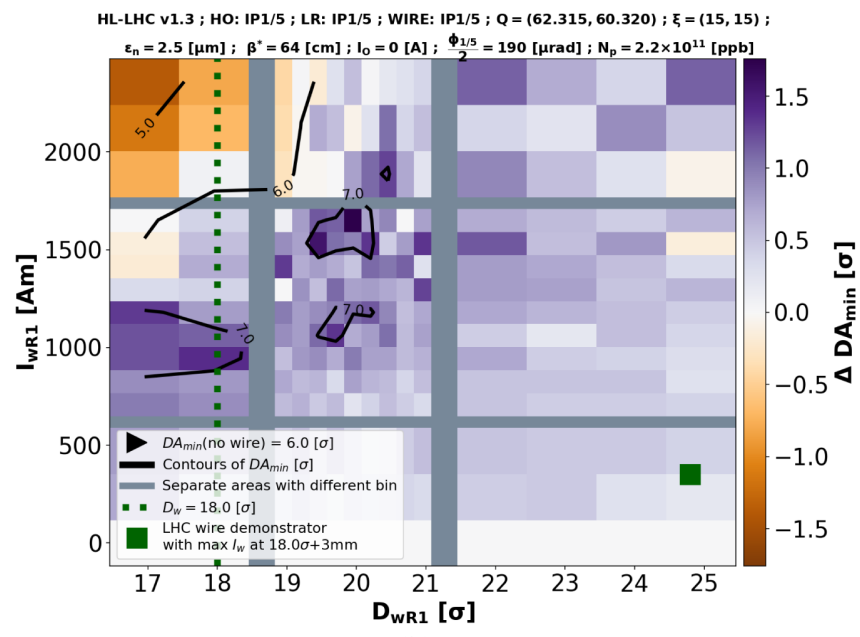

(b)

FIG. 10. HL-LHC at the start of luminosity leveling. (a) with half crossing angle at IP1 and IP5 $200 \mu \mathrm{rad}$ the $\Delta \mathrm{DA}_{\text {min }}$ for different wire configurations and (b) with half crossing angle at IP1 and IP5 $190 \mu \mathrm{rad}$ the $\Delta \mathrm{DA}_{\min }$ for different wire configurations.

different $D_{w}$ and $I_{w}$ are performed. The results for the improved ultimate and the improved nominal scenarios are presented in Figs. 10(a) and 10(b), respectively. Since the $\mathrm{DA}_{\min }$ (no wire) is larger than $6 \sigma$ for both machine configurations, the wire compensators are not needed at the start of collisions. However, almost all the configurations with $D_{w}$ above $17.4 \sigma$ [Fig. 10(a)] or $18 \sigma$ [Fig. 10(b)], which is the position of the tertiary collimators at the start of leveling for the ultimate and baseline scenarios, improve the $\mathrm{DA}_{\min }$ and guarantee a constant reduced crossing angle $\left(\Phi_{1 / 5}=380\right.$ or $\left.400 \mu \mathrm{rad}\right)$ through the leveling process.

\section{Integrated luminosity gain with the use of wire compensators}

Based on the results in Figs. 6 and $10\left(\mathrm{DA}_{\min }\right.$ close or above $6 \sigma$ with the assistance of dc wires), the crossing

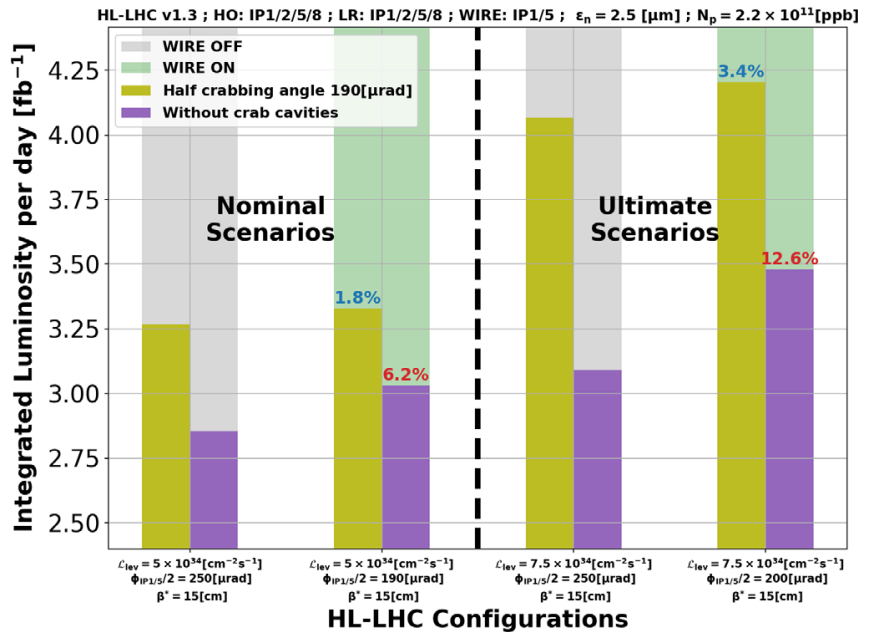

FIG. 11. The integrated luminosity per day for the current and improved nominal and ultimate scenarios of HL-LHC. angles during the luminosity leveling at the improved nominal and ultimate scenarios can be kept constant. Due to the smaller crossing angle at the improved scenarios, the luminosity leveling can be extended and the extra gain in integrated luminosity is presented in Fig. 11. With green shade are indicated the scenarios that need the dc wire to be operational (improved nominal and ultimate) and with gray shade are the current nominal and ultimate scenarios (no wire compensators). With the improved nominal scenario, the gain in integrated luminosity with the crab cavities on (190 $\mu \mathrm{rad}$ crabbing) is $\sim 2 \%$ over the existing nominal and $\sim 6 \%$ if the crab cavities are off. The increment is more significant with the improved ultimate scenario. The gain over the current ultimate (with $\mathrm{DA}_{\min }$ (no wire) $=4.7 \sigma$ ) is $\sim 3.5 \%$ with crab cavities and $\sim 12.5 \%$ without crab cavities.

\section{CONCLUSIONS}

In this paper it was demonstrated that for a variety of dc wire configurations, the detrimental effect from the long range beam beam interaction in different scenarios of the HL-LHC can be mitigated. Many of these good configurations respect the machine protection restrictions without sacrificing the beam lifetime. With all the best conditional configurations of the dc wire, the dynamic aperture of the PACMAN bunches is not affected and the area of good working points is enlarged. More specifically, for the baseline scenario where the minimum dynamic aperture is slightly above $6 \sigma$, the use of wire compensators can increase the $\mathrm{DA}_{\min }$ up to $0.65 \sigma$. This extra gain provide the margin for a flawless operation of the machine even in the presence of any unexpected detrimental effect like the strong electron cloud that was observed during the run II of the LHC. The wire compensator can also make the ultimate scenario $\left(\mathrm{DA}_{\min }\right.$ (no wire) $<6 \sigma$ ) fully operational with 
$\mathrm{DA}_{\min }>7.5 \sigma$. On top of that, with the use of wire compensators, the crossing angle of the nominal (baseline) and the ultimate scenarios can be reduced to $380 \mu \mathrm{rad}$ and $400 \mu \mathrm{rad}$ respectively. With these improved nominal and ultimate scenarios, the integrated luminosity per day is slightly increased with crab cavities and recovers half of the lost luminosity without the crab cavities. Because of the crossing angle reduction the crab cavities voltage can be also reduced without sacrificing the machine performance. Furthermore, the strength of the magnets (corrector) that generate the crossing bumps, the heat load and the integrated radiation that is mainly deposited in the final focus quadrupoles can be reduced. Finally, among the wire configurations that guarantee $\mathrm{DA}_{\min }$ close or above $6 \sigma$ for the improved scenarios $(190 \mu \mathrm{rad}$ and $200 \mu \mathrm{rad}$ half crossing angle) there are some that respect the collider protection restrictions and are technologically feasible. Therefore, the dc wires should be considered for future upgrades of the HL-LHC baseline.

\section{ACKNOWLEDGMENTS}

This research is supported by the HL-LHC project. The authors would like to thank Dr. Rogelio Tomas for his kind collaboration regarding the integrated luminosity calculations.

\section{APPENDIX: ELECTRIC AND MAGNETIC FIELDS}

The charge distributions that generate the BBLR kicks is described by a two dimensional Gaussian at the transverse plane and at the longitudinal one by a line distribution since $\sigma_{s} \gg \sigma_{\chi}$ with $\chi=x, y$. From such a charge distribution, the resultant transverse electromagnetic field satisfies the formula $B_{\phi}=-\beta_{l s} E_{r} / c$ in the lab rest frame. Therefore, the integrated Lorentz force experienced by a test particle in the weak beam with negligible transverse velocity is given by:

$$
\begin{aligned}
\int \vec{F}_{w e} d s & =\int q\left(\vec{E}+\vec{u}_{w e} \times \vec{B}\right) d s \Rightarrow \\
\int F_{w e} d s & =q\left(1+\beta_{w e} \beta_{l s}\right) \delta_{D}\left(s-s_{0}+u_{w e} t\right) \int E_{r} d s \\
& =q\left(1+\beta_{w e} \beta_{l s}\right) \frac{\delta_{D}\left(s-s_{0}\right)}{2} \int E_{r} d s \\
& =q\left(\mathcal{E}_{x}+\mathcal{E}_{y}\right) .
\end{aligned}
$$

$q$ is the electric charge of the test particle, $\delta_{D}$ is the Dirac delta function, $s=s_{0}+u_{w e} t, \delta_{D}(2 x) \equiv \delta_{D}(x) / 2, \beta_{l s}=$ $-u_{s t} / c, \beta_{w e}=u_{w e} / c$ and $c$ is the speed of light. The velocities $u_{s t}$ and $u_{w e}$ are measured in the lab rest frame and are the ones of the strong and weak bunches, respectively. A detailed derivation of the electromagnetic field that describe the beam-beam interactions is presented in $[32,53,54]$. Using these results the expressions for the electric $\left(\mathcal{E}_{\chi}\right)$ and magnetic $\left(\mathcal{B}_{\chi}\right)$ fields that include the relativistic term $1+\beta_{w e} \beta_{l s}$ and describe the BBLR interactions for round $\left(\sigma_{\chi}=\sigma_{\psi}=\sigma\right)$ and elliptical bunches $\left(\sigma_{\chi}>\sigma_{\psi}\right)$ are given by:

$$
\begin{gathered}
\mathcal{B}_{x}=\frac{\beta_{l s}}{c} \mathcal{E}_{y} \\
\mathcal{B}_{y}=-\frac{\beta_{l s}}{c} \mathcal{E}_{x} \\
\mathcal{E}=\mathcal{E}_{\psi}+\mathrm{i} \mathcal{E}_{\chi}=\mathcal{L} f \delta_{D}\left(s-s_{0}\right) \\
\mathcal{L}= \begin{cases}\frac{N_{p} q\left(1+\beta_{w e} \beta_{l s}\right)}{4 \epsilon_{0} \pi} & \text { for } \sigma_{\chi}=\sigma_{\psi}=\sigma \\
\frac{N_{p} q\left(1+\beta_{w e} \beta_{l s}\right)}{4 \epsilon_{0} \sqrt{\pi} \Delta_{\chi}} & \text { for } \sigma_{\chi}>\sigma_{\psi}\end{cases}
\end{gathered}
$$

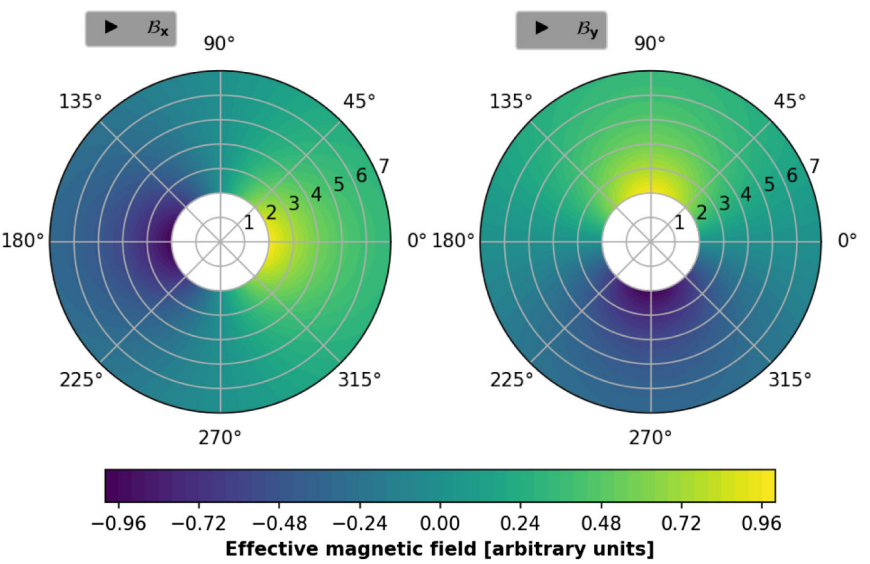

(b)

FIG. 12. Transverse magnetic field from: (a) a wire compensator and (b) a charge distribution with $\sigma_{x}>\sigma_{y}$. 


$$
f= \begin{cases}\frac{1}{\pi} \frac{\psi+\tilde{\psi}+\mathrm{i}(\chi+\tilde{\chi})}{(\chi+\tilde{\chi})^{2}+(\psi+\tilde{\psi})^{2}}\left(1-\operatorname{Exp}\left[-\frac{(\chi+\tilde{\chi})^{2}+(\psi+\tilde{\psi})^{2}}{2 \sigma^{2}}\right]\right) & \text { for } \sigma_{\chi}=\sigma_{\psi}=\sigma \\ \frac{1}{\sqrt{\pi} \Delta_{\chi}} \operatorname{Exp}\left[-\left(\frac{\chi+\tilde{\chi}+\mathrm{i}(\psi+\tilde{\psi})}{\Delta_{\chi}}\right)^{2}\right]\left(\operatorname{Erf}\left[\frac{(\psi+\tilde{\psi}) \sigma_{\chi}^{2}-\mathrm{i}(\chi+\tilde{\chi}) \sigma_{\psi}^{2}}{\sigma_{\chi} \sigma_{\psi} \Delta_{\chi}}\right]+\operatorname{Erf}\left[\frac{\mathrm{i}(\chi+\tilde{\chi})-(\psi+\tilde{\psi})}{\Delta_{\chi}}\right]\right) & \text { for } \sigma_{\chi}>\sigma_{\psi}\end{cases}
$$

where for round bunches $\left(\sigma_{\chi}=\sigma_{\psi}=\sigma\right) \chi=x$ and $\psi=y$ while for elliptical ones $\left(\sigma_{\chi}>\sigma_{\psi}\right) \chi=x, y$ and $\psi=x$ if $\chi=y$ or $\psi=y$ if $\chi=x$. The symbols $(\tilde{\chi}, \tilde{\psi})$ represent the transverse position of the weak bunch measured from the center of the strong bunch, where $(\chi, \psi)$ is the transverse position of the test particle measured from the center of the weak bunch, $\epsilon_{0}$ is the vacuum permittivity, $\Delta_{\chi}=$ $\sqrt{2\left(\sigma_{\chi}^{2}-\sigma_{\psi}^{2}\right)}$ and $\operatorname{Er} f[\Xi]$ is the error function with $\Xi$ a complex number.

The magnetic field $\left(B_{w}\right)$ generated by a wire compensator of length $L_{w}$ can be calculated with the use of the BiotSavart law [55]. However, since the wire length (a few meters long) is quite larger than the distance of the weak beam from the wire (less than a few centimeters), the magnetic field of an infinite long wire can be used. Thus, the integrated magnetic field $\mathcal{B}_{w}$ in complex form (that first developed in [31]) is written as:

$$
\begin{array}{r}
\mathcal{B}_{w}=\mathcal{B}_{w y}+\mathrm{i} \mathcal{B}_{w x}=\frac{\mu_{0} I_{w}}{2 \pi} f_{w} \delta_{D}\left(s-s_{0}\right), \\
f_{w}=\frac{1}{\mathcal{Z}_{r}+\mathcal{Z}_{w b}}=\sum_{u=0}^{\infty} \frac{(-1)^{u}}{\mathcal{Z}_{w b}^{u+1}} \mathcal{Z}_{r}^{u}=\sum_{u=0}^{\infty} c_{u} \mathcal{Z}_{r}^{u},
\end{array}
$$

where $I_{w}=\mathcal{J}_{w} L_{w}$ is the integrated current, $\mathcal{J}_{w}$ is the wire current, the $\mathcal{Z}_{r}=x+\mathrm{i} y$ is the test particle position measured from the weak beam and $\mathcal{Z}_{w b}=x_{w b}+\mathrm{i} y_{w b}$ is the position of the weak beam measured from the wire. Since $\mathcal{Z}_{w b}$ must be quite larger that the $\mathcal{Z}_{r}$, the function $f_{w}$ can be also expressed in a multipolar series as shown in Eq. (A3b) with multipole strength $c_{u}=\frac{(-1)^{u}}{\mathcal{Z}_{w b}^{u+1}}$.

The magnetic field generated by a bunch in the strong beam $(\mathcal{B})$ and the one generated by a wire compensator $\left(\mathcal{B}_{w}\right)$ are quite similar away from their sources. This can be seen in Figs. 12 where the magnetic fields at distances larger than $2 \sigma$ from their sources $\left(\sqrt{(\chi+\tilde{\chi})^{2}+(\psi+\tilde{\psi})^{2}} \geq 2 \sigma\right.$ and $\left.\sqrt{\operatorname{Re}\left[\mathcal{Z}_{r}+\mathcal{Z}_{w b}\right]^{2}+\operatorname{Im}\left[\mathcal{Z}_{r}+\mathcal{Z}_{w b}\right]^{2}} \geq 2 \sigma\right)$ are plotted. The $\mathcal{B}_{w x}$ and $\mathcal{B}_{w y}$ are shown in Fiq. 12(a) while the $\mathcal{B}_{x}$ and $\mathcal{B}_{y}$ for a charge distribution with $\sigma_{x}>\sigma_{y}$ can be seen in Fig. 12(b).

[1] O. S. Brning, P. Collier, P. Lebrun, S. Myers, R. Ostojic, J. Poole, and P. Proudlock, LHC Design Report, CERN Yellow Reports: Monographs (CERN, Geneva, 2004).
[2] J. Irwin, Diffusive losses from SSC particle bunches due to long-range beam-beam interactions, Technical Report No. SSC-233, SSC, Berkeley, CA, 1989.

[3] N. Gelfand, C. Johnstone, T. Sen, and W. Wan, Effect of the beam-beam interactions on the dynamic aperture of the LHC at collision, in IEEE Particle Accelerator Conference (PAC 99) (IEEE, New York, 1999), Vol. 3, pp. 1677-1679.

[4] Y. Papaphilippou and F. Zimmermann, Weak-strong beambeam simulations for the large hadron collider, Phys. Rev. ST Accel. Beams 2, 104001 (1999).

[5] Y. Papaphilippou and F. Zimmermann, Estimates of diffusion due to long-range beam-beam collisions, Phys. Rev. ST Accel. Beams 5, 074001 (2002).

[6] T. Sen, B. Erdelyi, M. Xiao, and V. Boocha, Beam-beam effects at the fermilab tevatron: Theory, Phys. Rev. ST Accel. Beams 7, 041001 (2004).

[7] W. Fischer, R. Calaga, U. Dorda, A. C. Kabel, J.-P. Koutchouk, J. Qiang, V. H. Ranjibar, T. Sen, J. Shi, and F. Zimmermann, Observation of the Long-range Beam-beam Effect in RHIC and Plans for Compensation, Proc. 10th European Particle Accelerator Conf. (EPAC'06), Edinburgh, UK (JACoW Publishing, 2006), p. WEPCH104, https://accelconf.web.cern.ch/e06/PAPERS/ WEPCH104.PDF.

[8] V. Shiltsev and A. Valishev, Long-range beam-beam effects in the Tevatron, ICFA Mini-Workshop on BeamBeam Effects in Hadron Colliders (2013), https://doi.org/ 10.5170/CERN-2014-004.101.

[9] R. Calaga, W. Fischer, G. Robert-Demolaize, and N. Milas, Long-range beam-beam experiments in the relativistic heavy ion collider, Phys. Rev. ST Accel. Beams 14, 091001 (2011).

[10] F. Zimmermann, 10 years of wire excitation experiments in the CERN SPS, in Proceedings, ICFA Mini-Workshop on Beam-Beam Effects in Hadron Colliders (BB2013): CERN, Geneva, Switzerland, March 18-22 2013 (JACoW, Geneva, 2014) pp. 153-166, arXiv: 1410.3654.

[11] V. Shiltsev, Y. Alexahin, V. Kamerdzhiev, G. Kuznetsov, and K. Bishofberger, Experimental demonstration of beambeam compensation by tevatron electron lenses and prospects for the LHC (2007), pp. 728-732.

[12] B. Erdelyi and T. Sen, Compensation of beam-beam effects in the Tevatron with wires, Technical Report No. FERMILAB-TM-2268-AD, 2004.

[13] J.-P. Koutchouk, Principle of a correction of the long-range beam-beam effect in LHC using electromagnetic lenses, CERN Technical Report No. LHC-PROJECT-NOTE-223, 2000; revised version number 1 submitted on 2000-11-20 10:24:42.

[14] G. Sterbini et al., First results of the compensation of the beam-beam effect with DC wires in the LHC, in 10th International Particle Accelerator Conference (JACoW, Geneva, 2019), p. WEYYPLM3. 
[15] A. Poyet, S. Fartoukh, N. Fuster-Martinez, N. Karastathis, Y. Papaphilippou, M. Pojer, S. Redaelli, A. Rossi, K. Skoufaris, M.S. Camillocci, and G. Sterbini, First experimental evidence of a beam-beam long-range compensation using wires in the Large Hadron Collider (to be published).

[16] A. Poyet, S. Fartoukh, N. Karastathis, Y. Papaphilippou, K. Skoufaris, and G. Sterbini, Numerical simulations of the DC wire prototypes in the LHC for enhancing the HL-LHC performances, in 10th International Particle Accelerator Conference (JACoW, Geneva, 2019), p. MOPMP052.

[17] A. Poyet, S. Fartoukh, N. Karastathis, Y. Papaphilippou, A. Rossi, G. Sterbini, and K. Skoufaris, Exploiting the beambeam wire demonstrators in the NextLHC Run 3, in 12th International Particle Accelerator Conference (JACoW, Geneva, 2021), p. MOPAB008.

[18] G. Apollinari, I. B. Alonso, O. Brning, P. Fessia, M. Lamont, L. Rossi, and L. Tavian, High-Luminosity Large Hadron Collider (HL-LHC): Technical Design Report V. 0.1, CERN Yellow Reports: Monographs (CERN, Geneva, 2017).

[19] I. B. Alonso and L. Rossi, HiLumi LHC Technical Design Report: Deliverable: D1.10, Technical Report No. CERNACC-2015-0140, 2015.

[20] E. Metral et al., Update of the HL-LHC operational scenarios for proton operation, Technical Report No. CERN-ACC-NOTE-2018-0002, 2018.

[21] L. Rossi and O. Brüning, Progress with the high luminosity LHC project at CERN, in 10th International Particle Accelerator Conference (JACoW, Geneva, 2019), p. MOYPLM3.

[22] N. Karastathis and Y. Papaphilippou, Beam-beam simulations for optimizing the performance of the Highluminosity Large Hadron Collider Proton Physics, Technical Report No. CERN-ACC-NOTE-2020-0026, 2020.

[23] The luminosity leveling in the HL-LHC is performed with the reduction of the $\beta^{*}$.

[24] R. B. Palmer, Energy scaling, crab crossing, and the pair problem, Report No. SLAC-PUB-4707, 1988, https:// searchworks.stanford.edu/view/11072982.

[25] R. Calaga, R. Tomas, and F. Zimmermann, LHC crabcavity aspects and strategy, Technical Report No. bNL90757-2010-CP, 2010.

[26] R. Calaga, Crab cavities for the high-luminosity LHC, in Proc. of International Conference on RF Superconductivity (SRF'17), Lanzhou, China, 2017, International Conference on RF Superconductivity (JACoW, Geneva, Switzerland, 2018), paper THXA03, pp. 695-699, http:// jacow.org/srf2017/papers/thxa03.pdf.

[27] M. Giovannozzi, Proposed scaling law for intensity evolution in hadron storage rings based on dynamic aperture variation with time, Phys. Rev. ST Accel. Beams 15, 024001 (2012).

[28] D. Pellegrini, F. Antoniou, G. Arduini, S. Fartoukh, G. Iadarola, N. Karastathis, S. Papadopoulou, Y. Papaphilippou, and G. Sterbini, Incoherent beam-beam effects and lifetime optimisation, in Proceedings, 2017 Workshop on LHC Beam Operations, Evian, CERN (CERN, Evian Les Bains, France, 2019).

[29] G. Arduini, O. Brning, F. Cerutti, R. de Maria, S. Fartoukh, M. Fitterer, G. Iadarola, D. Kaltchev, N. Karastathis,
A. Patapenka, D. Pellegrini, A. Poyet, A. Rossi, L. Rossi, K. Skoufaris, G. Sterbini, R. Tomas, and A. Valishev, Long range beam-beam effects for HL-LHC, in Proceedings, 2018 LHC Performance Workshop, Chamonix (CERN, Chamonix, France, 2018).

[30] S. Kostoglou, F. Antoniou, I. Efthymiopoulos, G. Iadarola, N. Karastathis, S. Papadopoulou, Y. Papaphilippou, K. Paraschou, D. Pellegrini, G. Sterbini, and G. Trad, Luminosity and lifetime modeling and optimization, in Proceedings, Evian Workshop on LHC beam operation, CERN (CERN, Evian Les Bains, France, 2019).

[31] S. Fartoukh, A. Valishev, Y. Papaphilippou, and D. Shatilov, Compensation of the long-range beam-beam interactions as a path towards new configurations for the high luminosity LHC, Phys. Rev. ST Accel. Beams 18, 121001 (2015).

[32] M. Bassetti and G. A. Erskine, Closed expression for the electrical field of a two-dimensional Gaussian charge, CERN Technical Reports No. CERN-ISR-TH-80-06, ISR-TH-80-06, 1980.

[33] E. Keil, Beam-beam dynamics, Conf. Proc. C 9309206, 539 (1993).

[34] A. Bertarelli, Wire hw design for HL-LHC and integration, in Proceedings of the Wire Compensation WP2/WP13 Satellite meeting (Fermilab, USA, 2019).

[35] D. Kaltchev (private communication).

[36] H. Mais and C. Mari, Introduction to beam-beam effects https://doi.org/10.5170/CERN-1994-001.499.

[37] D. Kaltchev, Fourier coefficients of long-range beam-beam Hamiltonian via two-dimensional Bessel functions, in Proceedings, 9th International Particle Accelerator Conference (IPAC 2018): Vancouver, BC Canada (JACoW, Geneva, 2018), p. THPAK108.

[38] K. Skoufaris, G. Sterbini, and Y. Papaphilippou, Resonance driving terms and tune spread with amplitude driven a beam-beam long range kick and a dc wire kick, arXiv:2103.05914.

[39] D. Laurent, G. Hans, R. Ghislain, and F. Schmidt, The MAD-X Program (Methodical Accelerator Design) Version 5.05.02 Users Reference Manual (2019).

[40] R. De Maria et al., SixTrack V and runtime environment, Int. J. Mod. Phys. A 34, 1942035 (2019).

[41] S. Fartoukh et al., Combined ramp and telescopic squeeze, CERN Technical Report No. CERN-ACC-2020-0028, 2020.

[42] J. M. Jowett, Collision schedules and bunch filling schemes in the LHC, CERN Technical Report No. LHC-PROJECT-NOTE-179, 1999.

[43] All the filling schemes (standard, BCMS and 8b $+4 \mathrm{e}$ ) can be found in: https://espace.cern.ch/HiLumi/WP2/Wiki/HLLHC\%20Parameters.aspx.

[44] U. Dorda, Compensation of long-range beam-beam interaction at the CERN LHC, Ph.D. thesis (2008), presented on 02 Jun 2008.

[45] X. Buffat, W. Herr, N. Mounet, T. Pieloni, and S. White, Stability diagrams of colliding beams in the large hadron collider, Phys. Rev. ST Accel. Beams 17, 111002 (2014).

[46] S. Fartoukh, The sign of the LHC octupoles, CERN Technical Report No. CERN-ACC-SLIDES-2014-0113, 2012. 
[47] R. G. Ala, M. Brugger, F. Cerutti, S. Danzeca, A. Ferrari, S. Gilardoni, Y. Kadi, M. Kastriotou, A. Lechner, C. Martinella, O. Stein, Y. Thurel, A. Tsinganis, and S. Uznanski, LHC and HL-LHC: Present and future radiation environment in the high-luminosity collision points and RHA implications, IEEE Trans. Nucl. Sci. 65, 448 (2018).

[48] J. Laskar, C. Froeschl, and A. Celletti, The measure of chaos by the numerical analysis of the fundamental frequencies. application to the standard mapping, Physica D: Nonlinear Phenomena 56, 253 (1992).

[49] A. A. N. Kolmogorov, Preservation of conditionally periodic movements with small change in the hamilton function, in Stochastic Behavior in Classical and Quantum Hamiltonian Systems, edited by G. Casati and J. Ford (Springer Berlin Heidelberg, Berlin, Heidelberg, 1979), pp. 51-56.

[50] J. Moser, On invariant curves of area-preserving mappings of an annulus, Nachr. Akad. Wiss. Gttingen, II 1 (1962).
[51] A. B. Givental, B. A. Khesin, J. E. Marsden, A. N. Varchenko, V. A. Vassiliev, O. Y. Viro, and V. M. Zakalyukin, Proof of a theorem of a. n. kolmogorov on the invariance of quasi-periodic motions under small perturbations of the hamiltonian, in Collected Works: Representations of Functions, Celestial Mechanics and KAM Theory, 1957-1965 (Springer Berlin Heidelberg, Berlin, Heidelberg, 2009), pp. 267-294.

[52] J. Laskar, Frequency map analysis of an Hamiltonian system, AIP Conf. Proc. 344, 130 (1995).

[53] K. Hirata, H. W. Moshammer, and F. Ruggiero, A symplectic beam-beam interaction with energy change, KEK ReportNo. KEK-92-117, 1992, https://cds.cern.ch/record/ 243013.

[54] G. Iadarola, R. De Maria, and Y. Papaphilippou, 6D beambeam interaction step-by-step, Technical Report No. CERN-ACC-NOTE-2018-0023, 2017.

[55] J. D. Jackson, Classical Electrodynamics, 3rd ed. (Wiley, New York, NY, 1999). 